\title{
Comparison of Time Series and Random-Vibration Theory Site-Response Methods
}

\author{
by Albert R. Kottke and Ellen M. Rathje
}

\begin{abstract}
The random-vibration theory (RVT) approach to equivalent-linear siteresponse analysis is often used to simulate site amplification, particularly when large numbers of simulations are required for incorporation into probabilistic seismic-hazard analysis. The fact that RVT site-response analysis does not require the specification of input-time series makes it an attractive alternative to other site-response methods. However, some studies have indicated that the site amplification predicted by RVT site-response analysis systematically differs from that predicted by time-series approaches. This study confirms that RVT site-response analysis predicts site amplification at the natural site frequencies as much as 20\%-50\% larger than time-series analysis, with the largest overprediction occurring for sites with smaller natural frequencies and sites underlain by hard rock. The overprediction is caused by an increase in duration generated by the site response, which is not taken into account in the RVT calculation. Correcting for this change in duration brings the RVT results within $20 \%$ of the timeseries results. A similar duration effect is observed for the RVT shear-strain calculation used to estimate the equivalent-linear strain-compatible soil properties. An alternative to applying a duration correction to improve the agreement between RVT and timeseries analysis is the modeling of shear-wave velocity variability. It is shown that introducing shear-wave velocity variability through Monte Carlo simulation brings the RVT results consistently within $\pm 20 \%$ of the time-series results.
\end{abstract}

\section{Introduction}

Local soil conditions influence the characteristics of earthquake ground shaking and these effects must be taken into account when specifying ground-shaking levels for seismic design. These effects are often quantified via siteresponse analysis, which involves the propagation of earthquake motions from the base rock through the overlying soil layers to the ground surface. Site-response analysis provides surface acceleration-time series, surface accelerationresponse spectra, and/or spectral-amplification factors based on the dynamic response of the local soil conditions.

In most cases, 1D site-response analysis is performed to assess the effect of soil conditions on ground shaking because vertically propagating, horizontally polarized shear waves dominate the earthquake ground-motion wave field. The most common technique for site-response simulations is equivalent-linear analysis using time-series input motions. The motion-to-motion variability between input-time series requires that simulations be performed using a suite of time series to generate a stable estimate of the expected site response. A potential alternative is random-vibration theory (RVT), which uses the same equivalent-linear wave-propagation approach, but allows for the calculation of the mean response with only one simulation. RVT-based site-response analyses are com- monly used for site-response calculations that are incorporated into probabilistic seismic-hazard analysis for nuclear power plants (Regulatory Guide 1.208; Nuclear Regulatory Commission [NRC], 2007) because in these cases, numerous site-response simulations are performed to account for a suite of scenario events and the variability in the site characteristics (i.e., depth to bedrock, shear-wave velocity profile, and nonlinear properties).

Extensive validation of RVT within the context of stochastic simulations of ground motion has been performed within the seismological community; examples of such work include Hanks and McGuire (1981), Boore (1983), and McGuire et al. (1984). Within the context of site-response analysis, fewer comparisons between RVT and time-series analyses have been conducted. Electric Power Research Institute (EPRI) (1993) compared RVT site-response predictions with recordings from a number of downhole arrays. The study focused on comparisons between observed surface motions and the surface motions computed by various siteresponse methods, including an RVT method. The study found that the RVT method did just as well as the time-series methods in predicting the surface motions. Rathje and Ozbey (2006) compared the results from site-specific site-response 
analyses using time series and RVT methods. This study generally showed a favorable comparison between RVT and time-series analyses, but in several cases the RVT analysis systematically predicted larger amplification at the site period. The study by Rathje and Ozbey (2006) was the first in the literature to identify a potential overprediction of site amplification by RVT, but the analyses were limited to only a single, hypothetical soil site. This paper expands upon the work of Rathje and Ozbey (2006) to better elucidate the differences between RVT and time-series site-response analyses.

In this paper a comprehensive comparison of the time series (TS) and RVT-based site-response techniques is performed for a range of site conditions (i.e., shallow soil to very deep soil) and over a range of input intensities that induce different levels of nonlinearity. The site conditions and input intensities used in the comparisons are selected to span the range of site conditions for which site-response analyses are performed. The goals of these comparisons are to identify systematic differences between TS and RVT site-response analyses for deterministic site conditions and to explain the observed differences. As such, this paper focuses on comparing two different site-response simulation techniques rather than comparing site-response simulations with site-response observations.

\section{Site-Response Methods}

Equivalent-linear (EQL) site-response analysis (Schnabel et al., 1972) uses frequency domain-transfer functions to model the 1D, linear-elastic wave propagation through a layered soil deposit. The method incorporates soil nonlinearity through the use of strain-compatible soil properties for each soil layer. The key to the EQL approach is the selection of soil properties, shear-modulus $(G)$, and damping $(D)$, for each soil layer that are consistent with the level of shear strain induced by the input rock motion. Development of strain-compatible properties requires an iterative approach in which the strains are computed, the properties are revised based on the strains, and revised strains are computed based on the updated properties. These iterations continue until the properties assigned to each layer in the wave-propagation analysis are consistent with the strains generated in each layer. The strain level used to select the strain-compatible properties is not the peak timedomain shear strain, but rather an effective shear strain $\left(\gamma_{\mathrm{eff}}\right)$ that is typically taken as about $65 \%$ of the peak value.

EQL wave-propagation analysis requires specification of an input rock motion. This input acceleration-time series is converted to the frequency domain using the Fast Fourier Transform (FFT). The resulting Fourier amplitude spectrum (FAS) is multiplied by the frequency domain-transfer function that represents wave propagation to the ground surface, and the FAS at the surface is converted to an accelerationtime series using the inverse FFT. Commonly a suite of three to seven input motions, selected and scaled to match a target response spectrum, is specified for use in this analysis because motion-to-motion variability induces different levels of nonlinearity and different responses.

To avoid selection and scaling of input acceleration-time histories and the computation time required to perform site-response analyses for multiple ground motions, the RVT approach can be used. A full description of the RVT approach can be found elsewhere (e.g., Silva et al. 1997; Boore, 2003; Rathje and Ozbey, 2006), but a summary is provided here. In an RVT site-response analysis, the input motion is characterized solely by the amplitude of the FAS and the groundmotion duration $\left(D_{\mathrm{gm}}\right)$. Because only the amplitudes of the FAS are known, without the associated phase angles, the specified FAS cannot be directly converted into an acceleration-time series. However, the FAS can be used to calculate peak time-domain parameters of motion, such as peak ground acceleration (PGA), through the use of extreme value statistics. In this approach, first the root mean square (rms) acceleration $\left(a_{\mathrm{rms}}\right)$ is computed from the FAS and the rms duration $\left(D_{\text {rms }}\right)$ using Parseval's theorem, as follows:

$$
a_{\mathrm{rms}}=\sqrt{\frac{2}{D_{\mathrm{rms}}} \int_{0}^{\infty}|A(f)|^{2} d f}=\sqrt{\frac{m_{0}}{D_{\mathrm{rms}}}},
$$

where $A(f)$ is the Fourier amplitude at frequency $f, m_{0}$ is the zero-th moment of the FAS, and $D_{\text {rms }}$ is taken as the groundmotion duration $\left(D_{\mathrm{gm}}\right)$ when using RVT to compute PGA. The $k$ th spectral moment of the FAS is defined by

$$
m_{k}=2 \int_{0}^{\infty}(2 \pi f)^{k}|A(f)|^{2} d f .
$$

To compute the expected maximum acceleration in the time domain ( $a_{\mathrm{max}}$, which represents PGA), $a_{\mathrm{rms}}$ is multiplied by a peak factor $\left(\mathrm{pf}=a_{\mathrm{max}} / a_{\mathrm{rms}}\right)$. Cartwright and LonguetHiggins (1956) derived an expression for the expected value of the peak factor for bandlimited white noise as

$$
\mathrm{pf}=\frac{a_{\mathrm{max}}}{a_{\mathrm{rms}}}=\sqrt{2} \int_{0}^{\infty} 1-\left[1-\xi \exp \left(-z^{2}\right)\right]^{N_{e}} d z
$$

where $\xi$ is the bandwidth and is defined as

$$
\xi=\sqrt{\frac{m_{2}^{2}}{m_{0} m_{4}}}
$$

The bandwidth varies from 0 to 1 and quantifies whether the motion is narrow- or broadband, with values approaching 1.0 for a narrowband signal. In equation (3), $N_{e}$ is the number of extrema and is defined as

$$
N_{e}=\frac{D_{\mathrm{gm}}}{\pi} \sqrt{\frac{m_{4}}{m_{2}}} .
$$

$N_{e}$ increases as the duration of the motion increases and as the signal becomes more broadband (i.e., smaller $\xi$ ).

To compute spectral acceleration rather than PGA using the RVT approach, the FAS is first multiplied by the transfer function of a single-degree-of-freedom oscillator before 
(a)

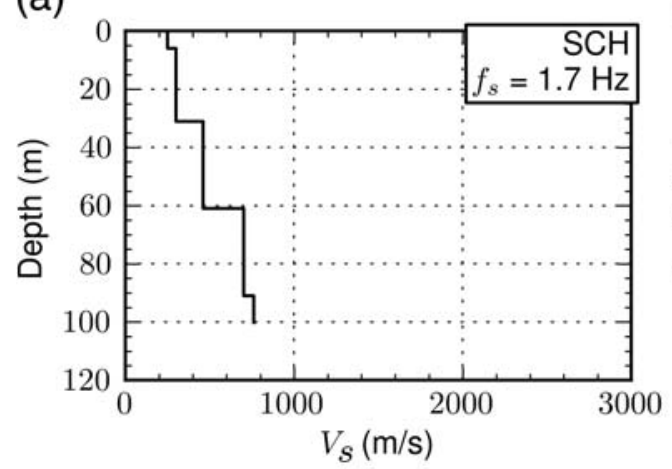

(c)

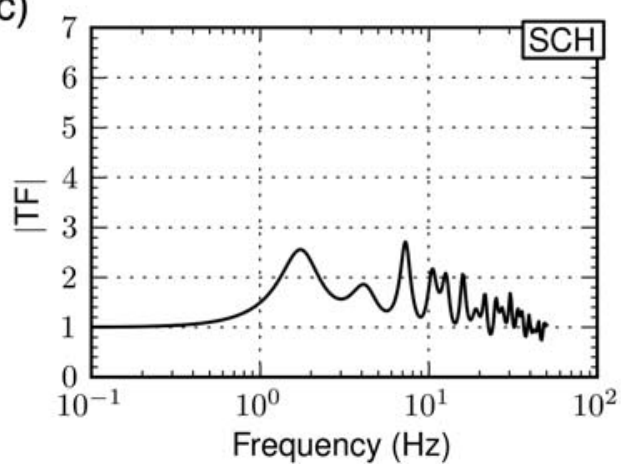

(b)

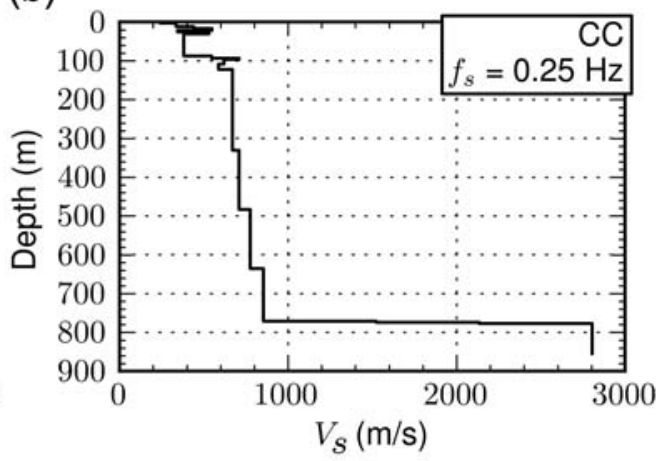

(d)

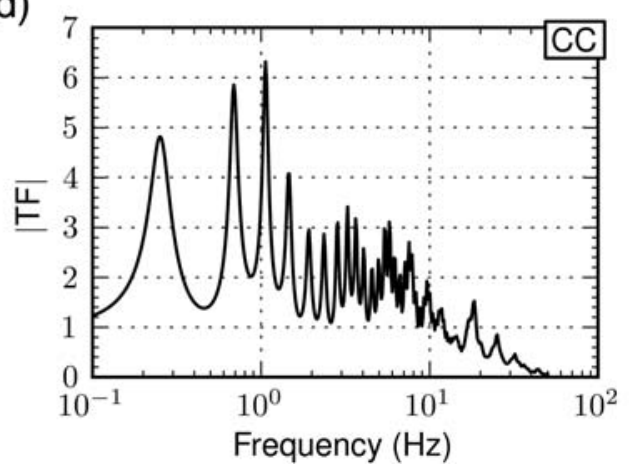

Figure 1. Shear-wave velocity $\left(V_{S}\right)$ profiles and theoretical site-transfer functions for the Sylmar County Hospital parking lot and Calvert Cliffs sites.

equations (1) through (5) are applied. Additionally, $D_{\text {rms }}$ must be modified from $D_{\mathrm{gm}}$, particularly for longer-period oscillators, because the oscillator response causes the motion to extend beyond the duration of the original motion. Boore and Joyner (1984) first proposed this oscillator correction as

$$
D_{\mathrm{rms}}=D_{\mathrm{gm}}+D_{o}\left(\frac{\gamma^{n}}{\gamma^{n}+\alpha}\right)
$$

where $\gamma=D_{\mathrm{gm}} / T_{o}, T_{o}$ is the oscillator natural period, $D_{o}$ is the oscillator duration given by $T_{o} / 2 \pi \zeta$, and $\zeta$ is the fraction of critical damping of the oscillator. Based on numerical simulations, Boore and Joyner (1984) proposed $n=3$ and $\alpha=1 / 3$. Liu and Pezeshk (1999) developed different values of $n$ and $\alpha$, whereas more recently Boore and Thompson (2012) developed an improved model for $D_{\text {rms }}$. The oscillator correction of Liu and Pezeshk (1999) is used in this study because it is adequate for the earthquake scenarios of engineering interest (Boore and Thompson, 2012).

Given that the input motion for RVT is specified by the amplitude of an FAS with no ability to reconstruct a time series, the RVT approach can only be applied to EQL siteresponse analysis in which the site response is modeled through frequency domain-transfer functions. The RVT approach cannot be applied to nonlinear analysis. For EQL analysis, the input rock FAS is multiplied by the amplitude of the site-transfer function, and the surface FAS is converted to a surface-response spectrum using the RVT methodology outlined above. The EQL soil properties are determined through iterations that incorporate RVT estimates of the peak shear strain. The RVT response spectrum represents the mean-response spectrum at the surface and thus provides in one analysis the same information as the mean from a suite of input motions from a TS analysis.

\section{Analyses Performed}

EQL site-response analyses using TS-input motions and using the RVT method are conducted for a range of site conditions. The sites are based on measured shear-wave velocity profiles from western North America (Sylmar County Hospital) and central and eastern North America (Calvert Cliffs), such that different fundamental site frequencies and regional characteristics can be investigated. Additionally, eight hypothetical site profiles are analyzed to better understand the observed trends. The shear-wave velocity profiles of the sites are described below. For each site, nonlinear-modulus reduction and damping curves are assigned based on soil type (plasticity index) and confining pressure using the empirical model of Darendeli (2001). The ground motions used in the analyses correspond to a magnitude 6.5 earthquake at a distance of $20 \mathrm{~km}$, as described below.

\section{Site Profiles}

The Sylmar County Hospital parking lot $(\mathrm{SCH})$ site is located in the San Fernando Valley of southern California and consists of $90 \mathrm{~m}$ of alluvium over rock (Fig. 1). 
Table 1

Characteristics of the Hypothetical Sites used in the Site-Response Analyses

\begin{tabular}{cccc}
\hline $\begin{array}{c}\text { Soil } \\
\text { Thickness }(\mathrm{m})\end{array}$ & $\begin{array}{c}V_{S}, \text { Soil } \\
(\mathrm{m} / \mathrm{s})\end{array}$ & $\begin{array}{c}\text { Site } \\
\text { Frequency }(\mathrm{Hz})\end{array}$ & $\begin{array}{c}V_{S}, \text { Rock } \\
(\mathrm{m} / \mathrm{s})\end{array}$ \\
\hline 10 & 400 & 10 & 1000,3000 \\
32 & 400 & 3.2 & 1000,3000 \\
100 & 400 & 1.0 & 1000,3000 \\
320 & 400 & 0.32 & 1000,3000 \\
\hline
\end{tabular}

Characteristic of southern California soil sites, there is not a significant impedance contrast as the profile encounters a shear-wave velocity typical of soft rock $(\sim 760 \mathrm{~m} / \mathrm{s})$. The site characteristics used in this study are based on measurements made by Gibbs et al. (1999). The theoretical linear-elastic transfer function for the $\mathrm{SCH}$ site, assuming $1 \%$ damping in all soil layers, is shown in Figure 1 and indicates a firstmode natural frequency of $1.7 \mathrm{~Hz}$ with an amplitude of about 2.5 .

The Calvert Cliffs (CC) site is located in Maryland, on the coast of the Chesapeake Bay. The site consists of alternating layers of sand and clay/silt to a depth of over $750 \mathrm{~m}$ (Fig. 1). The shear-wave velocity within the profile ranges from 400 to $900 \mathrm{~m} / \mathrm{s}$, while the rock underlying the soil column is late Paleozoic with a shear-wave velocity of almost $3000 \mathrm{~m} / \mathrm{s}$. The site characteristics used in the analyses are based on the site investigation reported by UniStar Nuclear (2007). The first-mode natural frequency is $0.25 \mathrm{~Hz}$, and the theoretical linear elastic-transfer function for $1 \%$ damping in the soil layers shows an amplitude of almost 5.0 at this frequency (Fig. 1). This large amplitude is a result of the large impedance contrast at the soil/rock interface.

Eight additional hypothetical shear-wave velocity profiles are created to better understand how the first-mode natural frequency and rock-impedance ratio influence the results.
Each hypothetical site is composed of a soil layer with a constant velocity underlain by a rock half-space. The shear-wave velocity and thickness of the soil layer are varied, as well as the shear-wave velocity of the rock half-space, as summarized in Table 1. The rock shear-wave velocities are selected to be representative of western North America (WNA) and central and eastern North America (CENA). The first-mode natural frequency of the sites ranges from $0.32 \mathrm{~Hz}$ for the deepest site to $10 \mathrm{~Hz}$ for the shallowest site. The theoretical site-transfer functions for a select group of the hypothetical sites are shown in Figure 2. For sites with a bedrock shearwave velocity of $1000 \mathrm{~m} / \mathrm{s}$ (Fig. 2a), the amplification at the first-mode frequency is approximately 3 and variations in soil thickness only shift the modal peaks. As the shear-wave velocity is increased to $3000 \mathrm{~m} / \mathrm{s}$ (Fig. 2b), the amplification at the first-mode frequency increases to approximately 8 .

\section{Input Ground Motions}

Input motions for the site-response analyses in this study are generated by stochastic simulation (e.g., Boore, 2003). The stochastic method first generates an FAS based on a seismological model that incorporates source, path, and site effects. This FAS can be used to generate input-time series for site-response analysis by scaling the FAS of a windowed time series of white noise or the FAS can be used directly as input into RVT site-response analysis. Because the time series generated by the stochastic method are consistent with a specified FAS and because RVT site-response methods require an input FAS, the stochastic method provides a good approach to generate input motions for comparisons of RVT and TS site-response analyses. However, a single stochastically simulated time series does not match the FAS exactly and therefore a suite of time series are required to match, on average, the FAS.
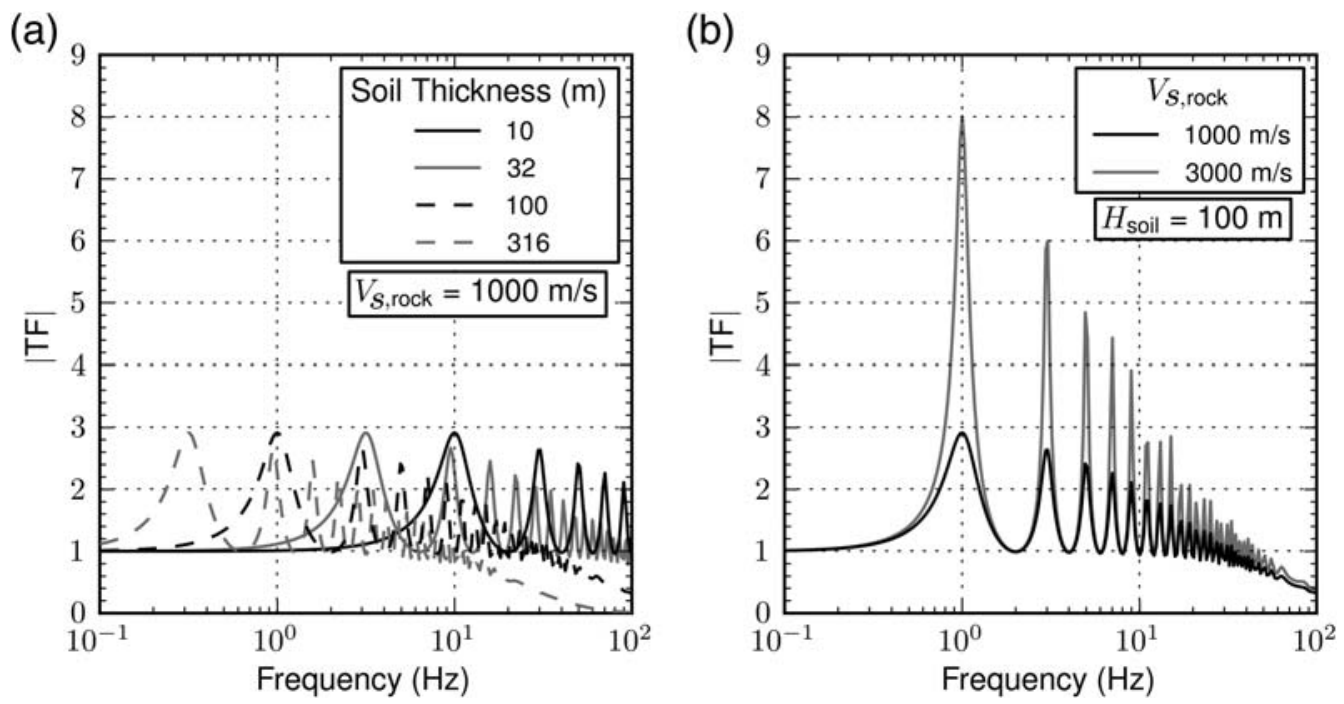

Figure 2. Theoretical site-transfer functions for selected hypothetical velocity profiles. 
Table 2

Selected Input Parameters from Boore (2005) used by SMSIM for the Calculation of the FAS shown in

Figure 3

\begin{tabular}{lc}
\hline \multicolumn{1}{c}{ Parameter } & \multicolumn{1}{c}{ Value } \\
\hline Source spectrum & Point source Brune $\omega$-squared \\
Stress drop, $\Delta \sigma(\mathrm{bar})$ & 80 \\
Site diminution, $\kappa(\mathrm{s})$ & 0.03 \\
Density of crust, $\rho\left(\mathrm{g} / \mathrm{cm}^{3}\right)$ & 2.8 \\
$V_{S}$ of crust, $\beta(\mathrm{km} / \mathrm{s})$ & 3.6 \\
$D_{\text {gm }}$ & Atkinson and Boore $(2006)$ \\
Crustal Amplification & Boore (2005) \\
\hline
\end{tabular}

The input motions for this study are based on a hypothetical $M_{\mathrm{w}} 6.5$ earthquake at a distance (R) of $20 \mathrm{~km}$ in WNA. The stochastic-input motions for this event are computed for a point source using the program SMSIM (Boore, 2005) and model parameters typical for shallow crustal events in WNA, as given by Boore (2005). The key model parameters are presented in Table 2 . The computed duration of the event for the RVT calculation $\left(D_{\mathrm{gm}}\right)$ is $6.80 \mathrm{~s}$. The resulting RVT-generated response spectrum and FAS for the seismological model are shown in Figure 3a,b. One hundred time series are generated from the seismological FAS and the resulting response spectra and FAS are shown in Figure 3c,d. The FAS and response spectra agree favorably between RVT and time series, demonstrating the consistency in the inputmotion specifications for RVT using stochastic simulations. All 100 simulated time series were used in the TS siteresponse analyses.

The level of variability in a suite of input-time series can influence the mean predictions of site response, such that this issue should be considered when comparing with RVT results. The logarithmic standard deviation of the response spectra $\left(\sigma_{\operatorname{lnSa}}\right)$ of the 100 time series is about 0.40 at $0.20 \mathrm{~Hz}$ and decreases to 0.15 at $100 \mathrm{~Hz}$. To better understand the influence of the variability in the suite of input-time series on the results from TS site-response analyses, the stochastic time series are modified to reduce their variability using two techniques. In the first technique, the amplitudes of each FAS are modified to match the seismological FAS. This technique is referred to as Fourier amplitude matching or FA matched. The FA matched motions are time series that agree very closely with the seismological FAS (Fig. 3f) and have some reduced variability in the response spectra particularly at longer periods (Fig. 3e). While the resulting motions may no longer have realistic characteristics in the time domain, these motions are used in this study to model minimal variability in the frequency domain. In the second technique, the response spectra of the 100 simulated time series are spectrally matched (SA matched) to the RVT response spectrum using RSPMATCH (Hancock, 2006). The spectral matching is performed from 0.2 to $30 \mathrm{~Hz}$, which results in the TS response spectra agreeing well over this frequency range but not as well outside of this range (Fig. $3 \mathrm{~g}$ ). While the response spectra show little variability for the spectrally matched motions, the FAS still contain variability (Fig. 3h).

\section{Comparison of Time Series and RVT Site-Response Results}

Time series and RVT site-response analyses are performed using the program Strata and used to calculate the amplification factor (AF $=S_{a \text {,soil }} / S_{a \text {,rock }}$, where $S_{a}$ is spectral acceleration). The TS and RVT approaches to EQL site-response analysis are compared using the median amplification factor predicted from a suite of time series $\left(\overline{\mathrm{AF}}_{\mathrm{TS}}\right)$ and the $\mathrm{AF}$ values predicted by $\mathrm{RVT}\left(\mathrm{AF}_{\mathrm{RVT}}\right) . \overline{\mathrm{AF}}_{\mathrm{TS}}$ is calculated as the mean value in logarithmic space (i.e., the geometric mean). In earthquake-engineering practice, the median value is commonly used, whereas RVT predicts the mean value. The comparisons in this study will focus on the median of the time series because little difference was observed between the mean and median values of AF computed from the suite of simulated time series. The comparison between the RVT and TS results are quantified by the ratio of the RVT amplification factor to the median TS amplification factor, defined as

$$
\alpha_{\mathrm{AF}}=\frac{\mathrm{AF}_{\mathrm{RVT}}}{\overline{\mathrm{AF}}_{\mathrm{TS}}} .
$$

The agreement between the TS and RVT analyses is quantified using $\alpha_{\mathrm{AF}}$ versus frequency, as well as the minimum $\alpha_{\mathrm{AF}}$ and the maximum $\alpha_{\mathrm{AF}}$ over all frequencies.

\section{Linear-Elastic Comparisons}

Time series and RVT site-response analyses are first performed for linear-elastic (LE) soil conditions. The TS and RVT amplification factors for the SCH site (Fig. 4) are very similar, with the RVT results falling within the range of the time series. The differences between the TS and RVT results are better shown through the $\mathrm{AF}_{\mathrm{RVT}} / \mathrm{AF}_{\mathrm{TS}}$ ratio $\left(\alpha_{\mathrm{AF}}\right) . \alpha_{\mathrm{AF}}$ for $\mathrm{SCH}$ varies between 0.95 and 1.1 with the maximum occurring at the site frequency of $1.7 \mathrm{~Hz}$ (Fig. 4). The TS and RVT amplification factors for the CC site (Fig. 4) again show the RVT results falling within the range of the time series, but now there are significant differences between the median TS and RVT results, particularly at the site frequency. $\alpha_{\mathrm{AF}}$ for CC varies from 0.8 to 1.3 with the maximum of about 1.3 occurring at the first-, second-, and third-mode natural frequencies (Fig. 4).

The comparisons using the $\mathrm{SCH}$ and $\mathrm{CC}$ sites show that the agreement between the RVT and TS methods depends on the site characteristics. To better understand the influence of the site characteristics on the differences between the RVT and TS results, LE analyses are performed using the hypothetical sites. The associated $\alpha_{\mathrm{AF}}$ are shown in Figure 5. These results show that the maximum $\alpha_{\mathrm{AF}}$ always occurs at a site frequency and that it increases as the site frequency 
(a)

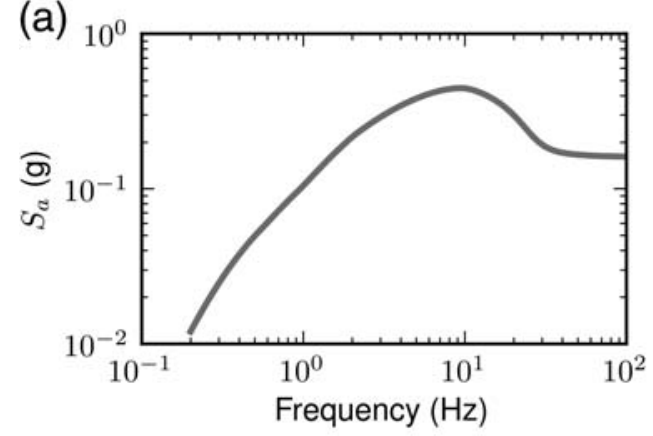

(c)

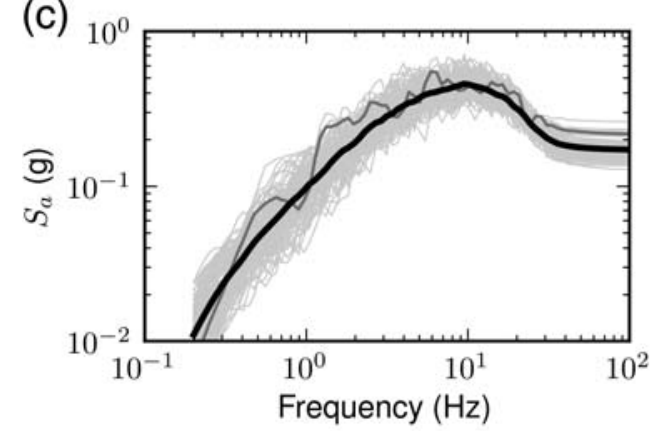

(e)

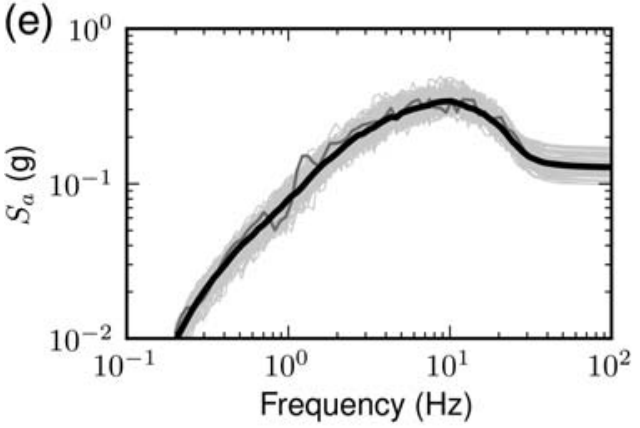

(g)

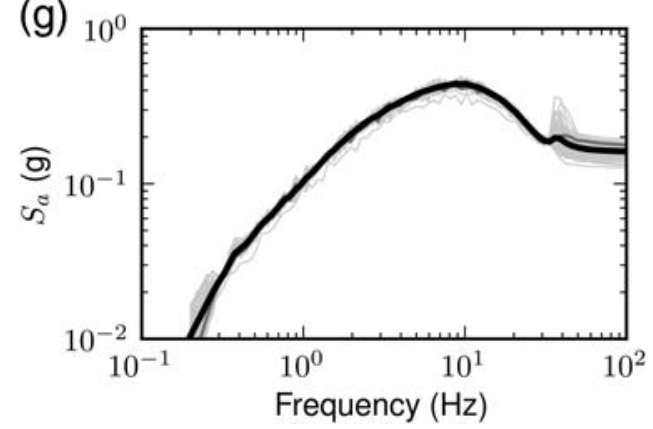

(b)

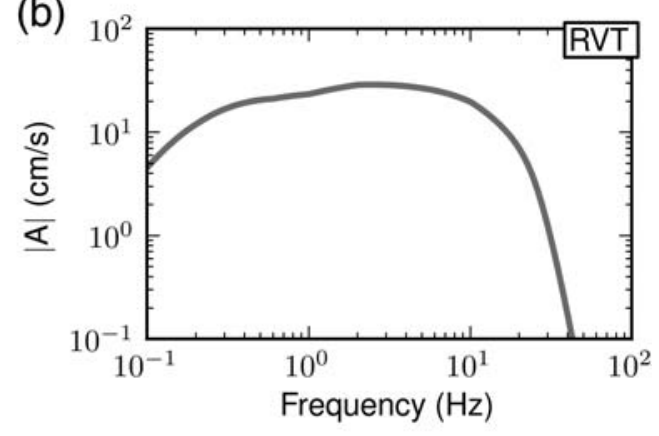

(d)
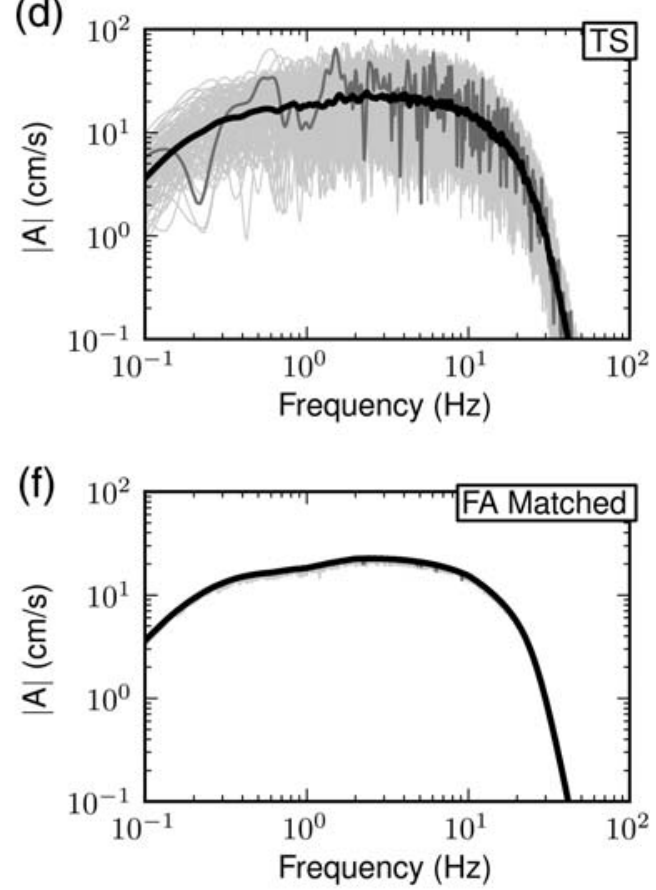

(h)

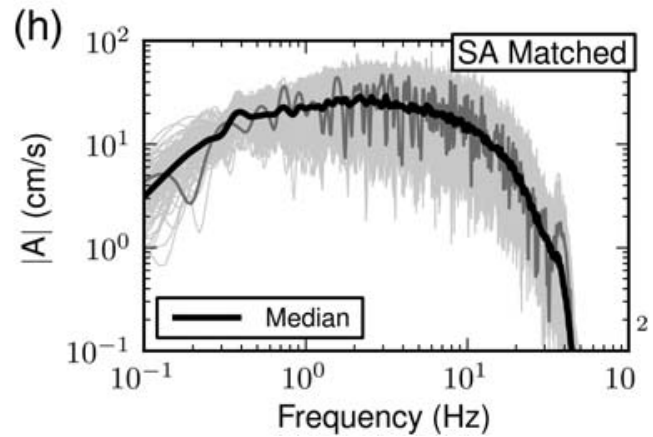

Figure 3. Acceleration-response spectra and Fourier amplitude spectra for (a and b) RVT-input motion, (c and d) stochastically simulated time-series motions, (e and f) simulated motions matched to the FAS, and ( $\mathrm{g}$ and $\mathrm{h}$ ) simulated motions spectrally matched to the RVT-response spectrum. The thin gray lines represent all time series generated. The thick gray line represents a single time series.

decreases (i.e., soil thickness, $H_{\text {soil }}$, increases) and as $V_{S, \text { rock }}$ increases. For example, as the site frequency decreases from $10 \mathrm{~Hz}\left(H_{\text {soil }}=10 \mathrm{~m}\right)$ to $0.32 \mathrm{~Hz}\left(H_{\text {soil }}=316 \mathrm{~m}\right)$, the maximum $\alpha_{\mathrm{AF}}$ increases from 1.05 to 1.2 for $V_{S \text {,rock }}=1000 \mathrm{~m} / \mathrm{s}$ and increases from 1.15 to 1.5 for $V_{S, \text { rock }}=3000 \mathrm{~m} / \mathrm{s}$. The influence of $V_{S \text {,rock }}$ on the results indicates that the height of the transfer function is also important, with large $V_{S \text {,rock }}$ associated with larger peaks in the transfer function (Figs. 1 and 2). These results demonstrate that the amplification predicted by RVT may be as much as $20 \%-50 \%$ larger than TS, which is significant. The potential under prediction is less severe, but still notable at $10 \%$ to $20 \%$.

One potential cause for the differences between RVT and TS analysis is the modification of the duration of the time 
(a)

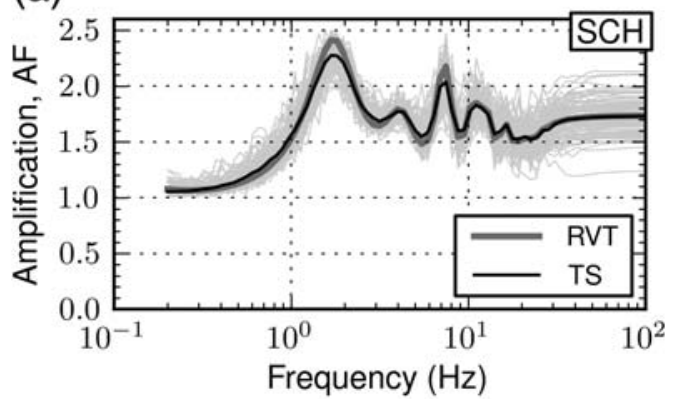

(c)

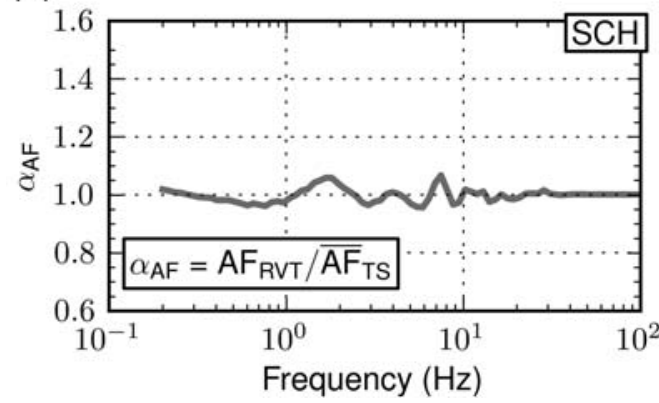

(b)

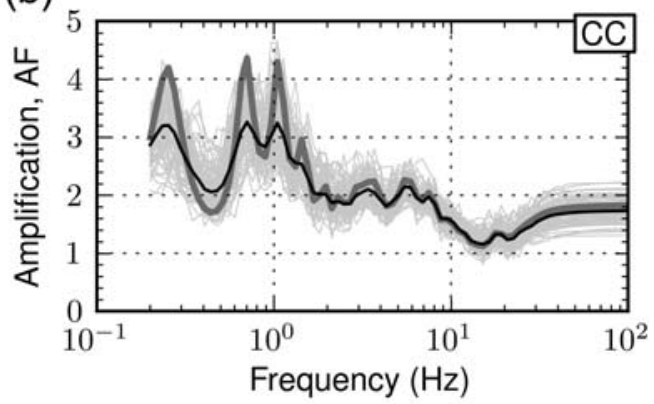

(d)

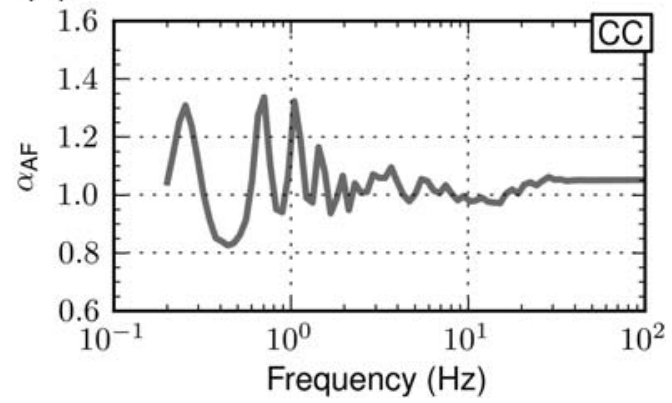

Figure 4. Amplification factors for TS and RVT analyses and $\alpha_{\mathrm{AF}}$ for the SCH and CC sites.

(a)

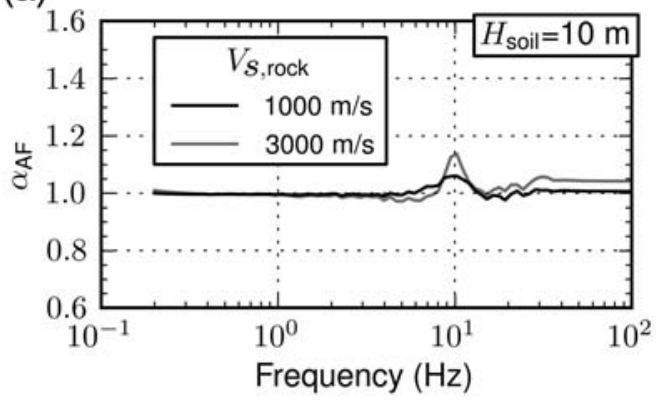

(c)

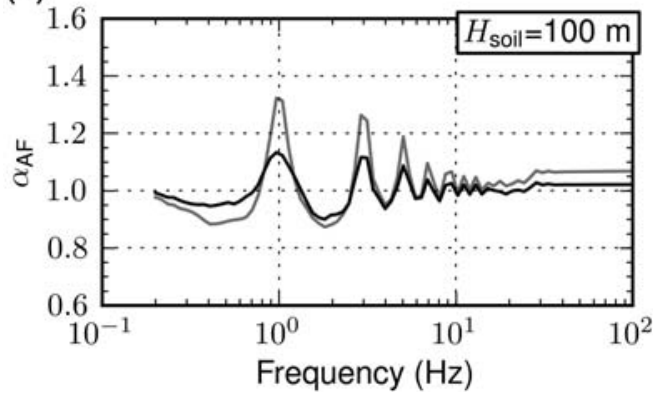

(b)

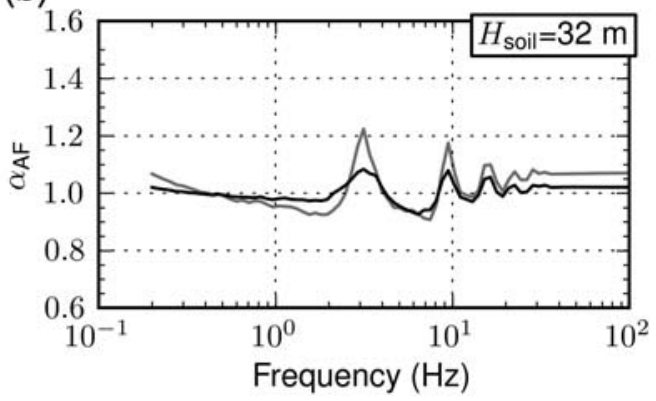

(d)

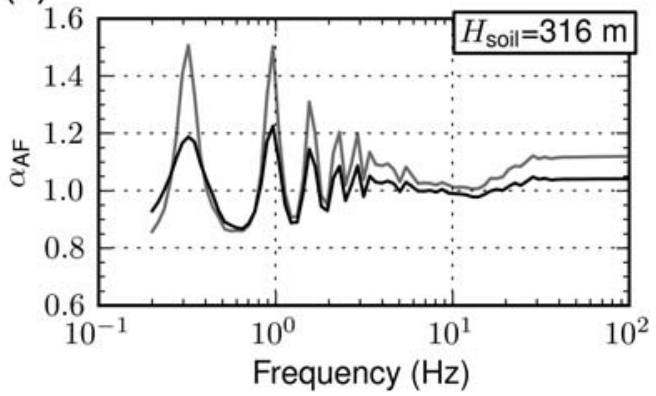

Figure 5. $\alpha_{\mathrm{AF}}$ for the eight hypothetical sites listed in Table 1 .

series due to the response of the site, which is ignored in the current implementation of RVT site response (i.e., the rock $D_{\mathrm{gm}}$ is used as the basis for $D_{\mathrm{rms}}$ in the RVT calculation of the surface response spectrum). As previously noted, the increase in duration due to the single-degree-of-freedom oscillator response must be included in the calculation of RVT response spectra. Thus it follows that the increase in duration due to the soil-column response should be taken into account. For example, Figure 6 shows an input accelerationtime history and the computed surface-time history for the $H_{\text {soil }}=316 \mathrm{~m}$ site with $V_{S \text {,rock }}=3000 \mathrm{~m} / \mathrm{s}$. The duration of the input motion, as defined based on $\mathrm{D}_{5-75}$ (i.e., the time interval between the occurrence of $5 \%$ and $75 \%$ of the Arias intensity of the acceleration-time history), is equal 
(a)

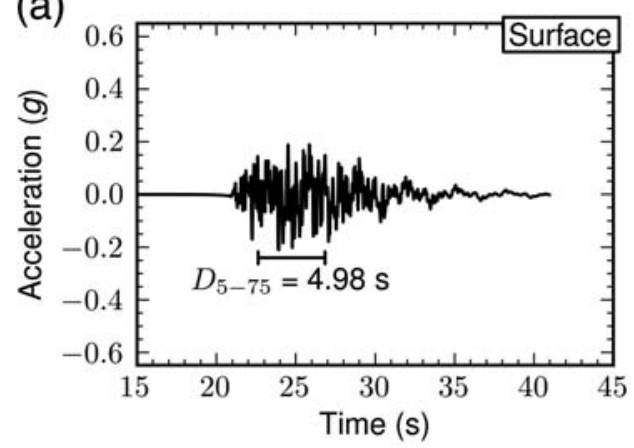

(c)

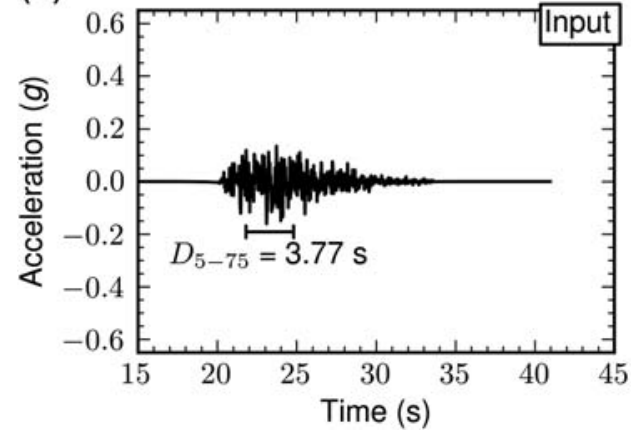

(b)

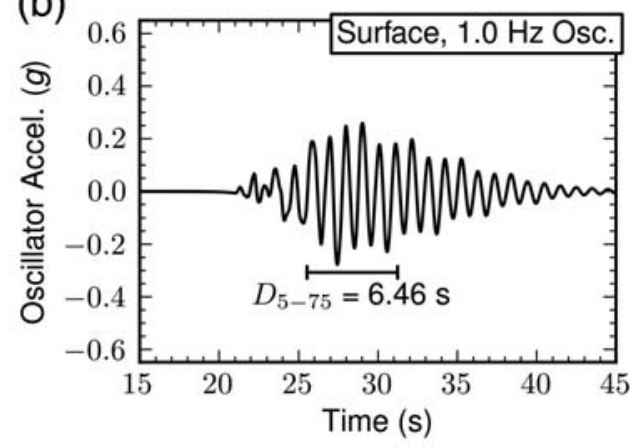

(d)

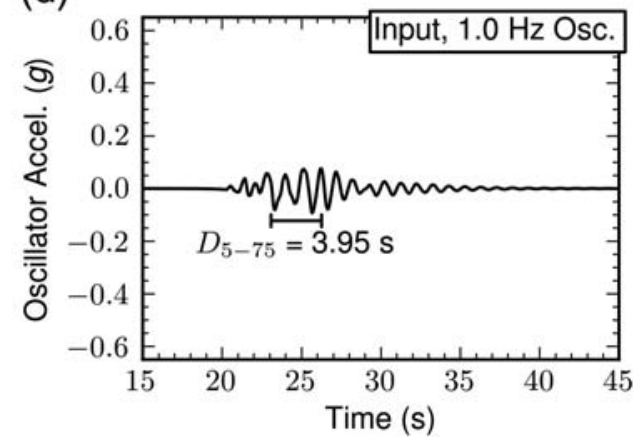

Figure 6. The influence of site response and oscillator response on ground-motion duration. Acceleration-time histories represent a TS-input motion, the computed surface motion for the $H_{\text {soil }}=316 \mathrm{~m}$ site for the TS-input motion, and the computed 1-Hz oscillator (5\% damping) responses for the input and surface motions.

to $3.77 \mathrm{~s}$. This duration is increased to $4.98 \mathrm{~s}$, a $32 \%$ increase, at the ground surface due to the site response. The duration effect is even more pronounced when considering the response of an oscillator subjected to these motions (Fig. 6). For an oscillator with a natural frequency of $1.0 \mathrm{~Hz}$, the duration increases from 3.95 to $6.46 \mathrm{~s}$, a $64 \%$ increase. An increase in duration for a given FAS results in a reduction in $a_{\mathrm{rms}}$ (equation 1), which then translates into smaller values of PGA and spectral acceleration. Ignoring these increases in duration generates RVT-computed spectral accelerations that are larger than the TS values.

To further investigate the modification in duration due to the site response, significant durations are computed for both the input-time series $\left(D_{5-75}^{\text {input }}\right)$ and surface-time series $\left(D_{5-75}^{\text {surface }}\right)$. Significant durations are computed for input- and surface-time series, as well as the oscillator responses at each of the oscillator frequencies for the input- and surface-time series. The ratio of $D_{5-75}^{\text {surface }}$ to $D_{5-75}^{\text {input }}$ is plotted versus oscillator frequency in Figure 7 for the surface motions computed from analysis of the $\mathrm{SCH}, \mathrm{CC}$, and eight hypothetical sites. The data show that for sites with first-mode natural frequencies greater than or equal to about $3 \mathrm{~Hz}$ ( $\mathrm{SCH}, H_{\text {soil }}=10 \mathrm{~m}$ and $32 \mathrm{~m}$ ) there is little change in the durations, with the duration ratios falling between 0.98 and 1.05 . However, as the natural frequency of a site decreases, differences between the inputmotion and surface-motion durations increase and these changes are most prevalent at the natural frequencies of the site. For example, when $H_{\text {soil }}=316 \mathrm{~m}\left(f_{\text {site }}=0.32 \mathrm{~Hz}\right)$, the ratio of $D_{5-75}^{\text {surface }}$ to $D_{5-75}^{\text {input }}$ at the natural frequency of the site is 1.2 for $V_{S, \text { rock }}$ of $1000 \mathrm{~m} / \mathrm{s}$ and 1.6 for $V_{S, \text { rock }}$ of $3000 \mathrm{~m} / \mathrm{s}$ (Fig. 7f). The change in duration is also influenced by the height of the transfer function, with the duration modified more when the bedrock shear-wave velocity is larger. This more significant increase in duration is caused by stronger multireflections in the soil column for larger $V_{S \text {,rock. The }}$ shapes of the curves in Figure 7 for duration are very similar to those in Figures 4 and 5 for amplification factor, suggesting that the overprediction in the RVT-amplification factor at a site's natural frequencies is related to the changes in the ground-motion duration due to site response.

The surface-response spectra and AF computed by RVT can be corrected to account for the change in duration due to the site response. Equation (1) indicates that $a_{\mathrm{rms}}$ is inversely proportional to the square root of $D_{\mathrm{rms}}$. Because the computed $\mathrm{AF}$ is directly proportional to $a_{\mathrm{rms}}$, the $\mathrm{AF}$ computed by RVT can be corrected by dividing by the square root of the observed duration ratio $\left(D_{5-75}^{\text {surface }} / D_{5-75}^{\text {input }}\right)$. The amplification factors computed by RVT are corrected using this methodology and the $\alpha_{\mathrm{AF}}$ computed from the corrected RVT results are shown in Figure 8. For the sites with high natural frequencies ( $\mathrm{SCH}, H_{\text {soil }}=10 \mathrm{~m}$ and $32 \mathrm{~m}$ ), the difference between the corrected and uncorrected $\alpha_{\mathrm{AF}}$ are insignificant because of the relatively small changes to the duration (Fig. 7). However, for the sites with smaller natural frequencies (CC, $H_{\text {soil }}=100 \mathrm{~m}$ and $316 \mathrm{~m}$ ), the duration correction reduces the largest $\alpha_{\mathrm{AF}}$ such that no value is above about 1.2. 
(a)

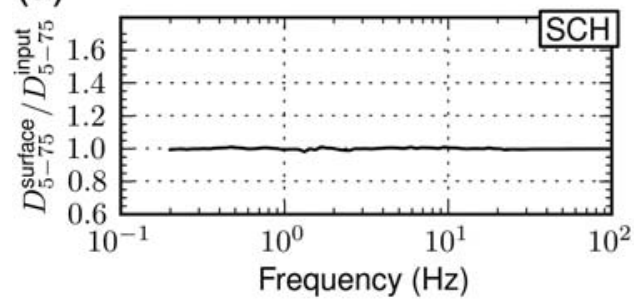

(c)

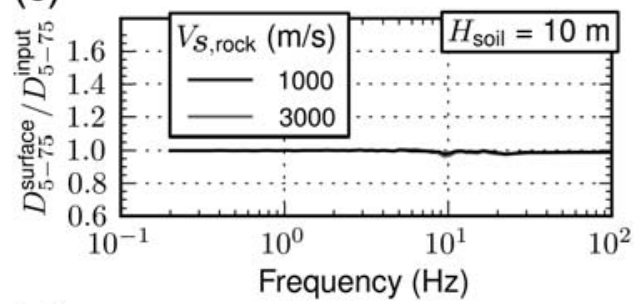

(e)

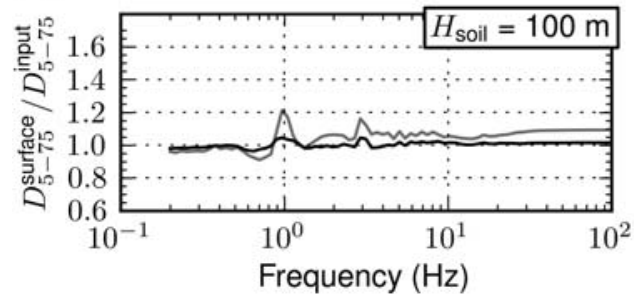

(b)

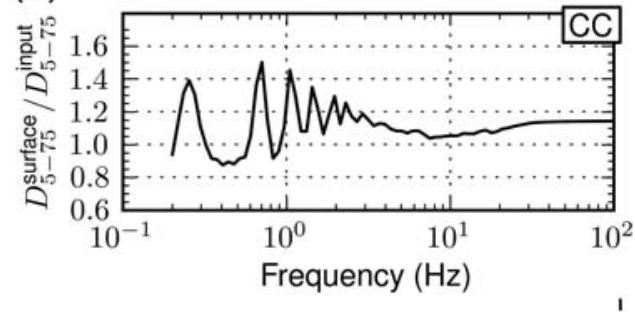

(d)

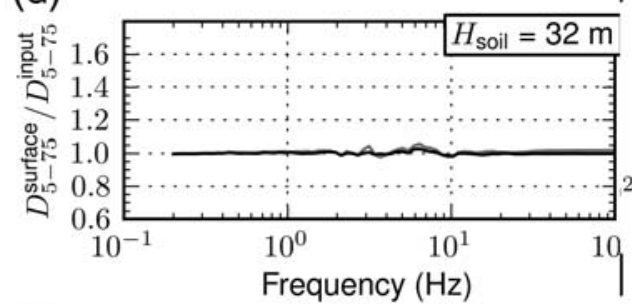

(f)

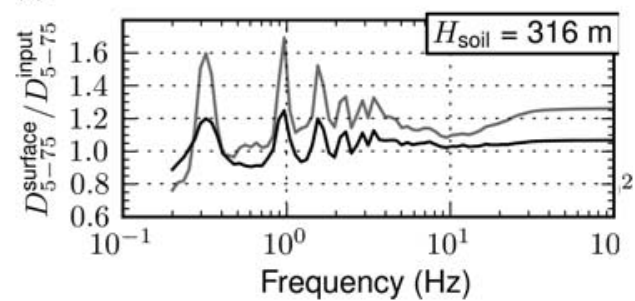

Figure 7. The ratio of the significant duration of the oscillator response of the surface motion to the significant duration of the oscillator response of the input motion.

Summarizing the results for all analyses performed, the computed values of minimum $\alpha_{\mathrm{AF}}$ and maximum $\alpha_{\mathrm{AF}}$ are plotted versus site frequency in Figure 9. Before the duration correction, the minimum $\alpha_{\mathrm{AF}}$ ranges from 0.82 to 0.98 with smaller values (more underprediction) occurring at smaller site frequencies. The duration correction has little effect on the minimum $\alpha_{\mathrm{AF}}$. For the maximum $\alpha_{\mathrm{AF}}$, the uncorrected values range from 1.05 to 1.5 with larger values (more overprediction) occurring at smaller site frequencies. The duration correction reduces the maximum $\alpha_{\mathrm{AF}}$ to below about 1.2 for all analyses performed and the sites with $V_{S, \text { rock }}=$ $1000 \mathrm{~m} / \mathrm{s}$ generally display maximum $\alpha_{\mathrm{AF}}$ values around 1.1 .

It is clear from the preceding analyses that site response influences ground-motion duration and that ignoring this change in duration in RVT analysis results in significant differences between RVT and TS estimates of site amplification. The duration correction for RVT explored here is based on computed values of $D_{5-75}$ of the input and surface motions from the TS analysis. However, the duration corrections developed by others to deal with duration changes due to oscillator response (e.g., Boore and Joyner, 1984; Liu and Pezeshk, 1999; Boore and Thompson, 2012) are based directly on the spectral amplitudes computed by RVT and TS simulations. An alternative to the approach explored here is to use the site-amplification results from the RVT and TS site-response analyses (e.g., Fig. 5) to directly compute the duration correction required to make the RVT and TS predictions match.

\section{Influence of Input-Motion Variability on RVT-TS Comparisons}

The influence of the variability in the input motions on the amplification factors computed by TS analysis is explored because the RVT site-response analysis uses a smooth FAS as input while the TS analysis uses multiple-time series that have motion-to-motion variability and nonsmooth FAS. Site-response simulations are conducted for all sites using LE soil conditions and the simulated, FA-matched and SA-matched time series (Fig. 3). The amplification factors computed for the hypothetical site with a thickness of $316 \mathrm{~m}$ and $V_{S, \text { rock }}=3000 \mathrm{~m} / \mathrm{s}$ are shown in Figure 10 for the three sets of input motions. Both the FA-matched and SA-matched motions generate moderately larger medianamplification factors than the original TS at the site frequencies, with the FA-matched motions providing the largest median response. The median-spectral ratio at the site frequency $(0.32 \mathrm{~Hz})$ is 4.1 for the original time series, 4.2 for the SA-matched motions, and 4.5 for the FA matched. The change in the median-amplification factor is caused by the matched motions generating almost no "very low" amplification factors (e.g., many motions have $\mathrm{AF}<3.0$ for the TS motions at the site frequency, whereas almost no motions fall 
(a)

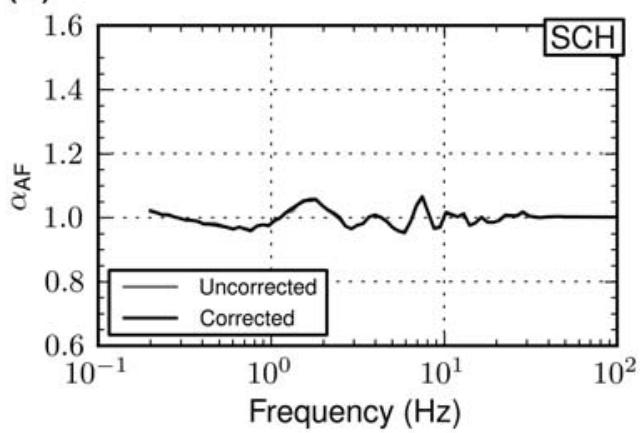

(c)

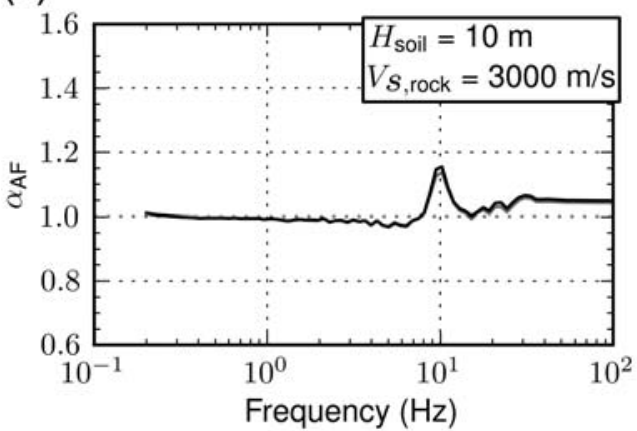

(e)

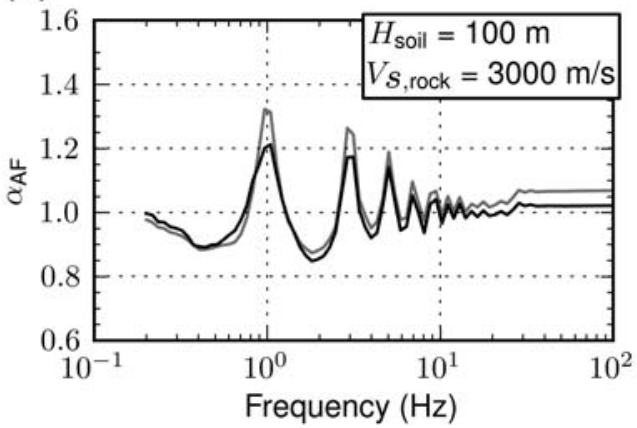

(b)

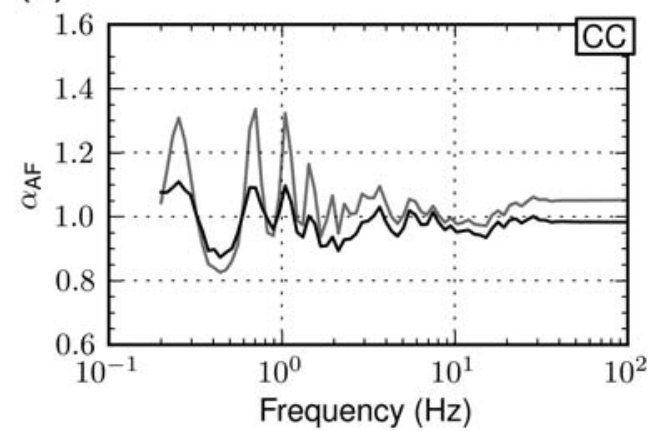

(d)

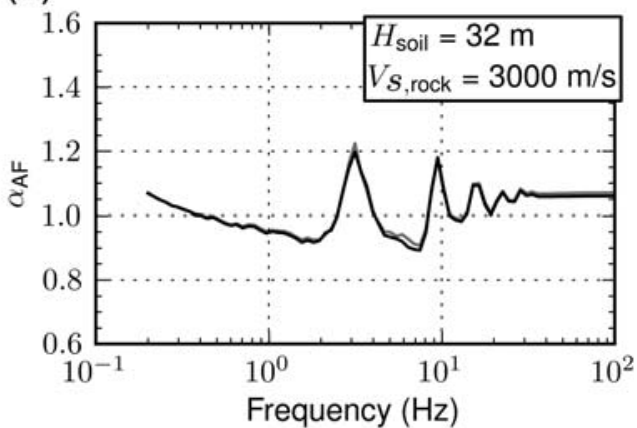

(f)

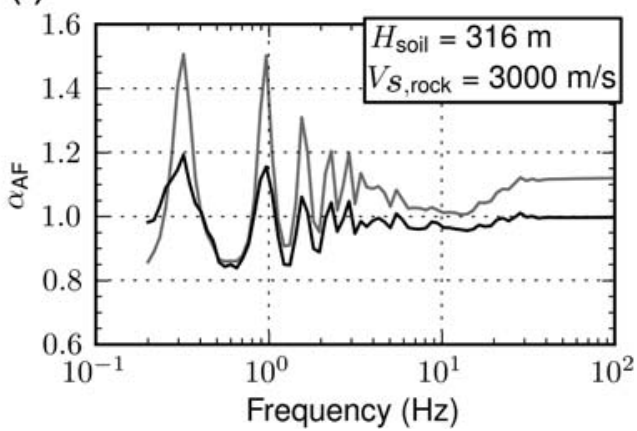

Figure 8. $\alpha_{\mathrm{AF}}$ computed with the uncorrected RVT results and duration-corrected RVT results for each site analyzed.
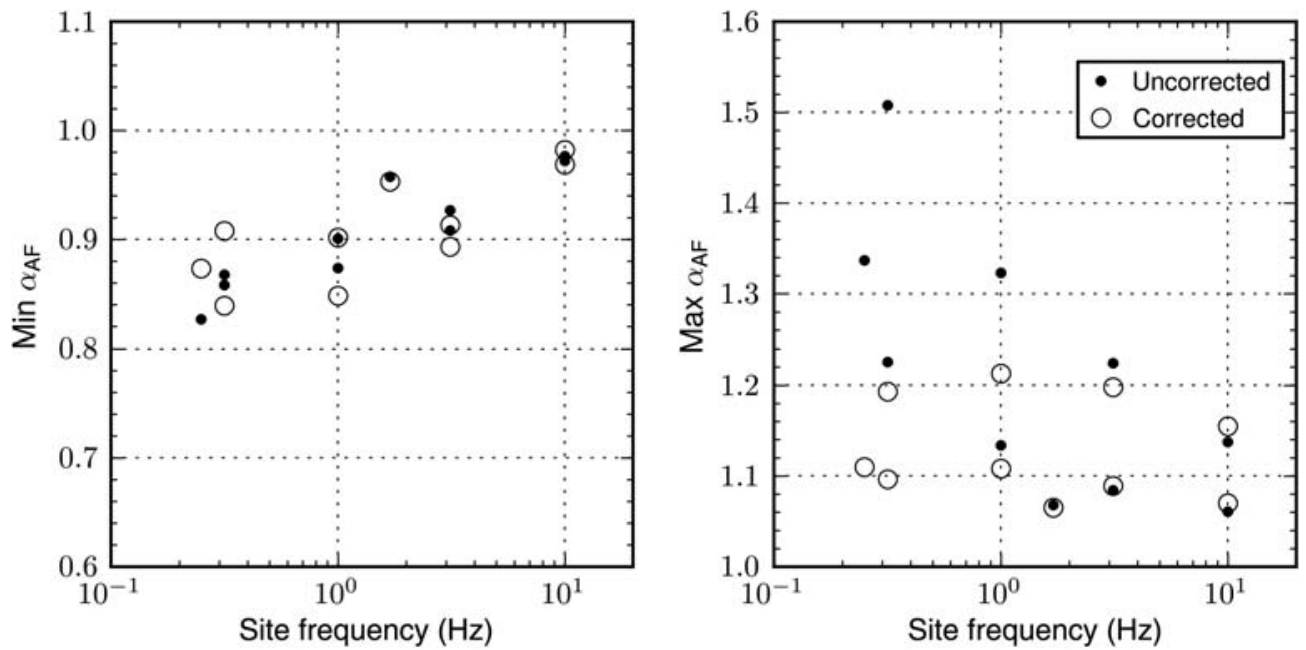

Figure 9. The maximum and minimum $\alpha_{\mathrm{AF}}$ as a function of site natural frequency for the uncorrected and duration-corrected RVT results. 
(a)

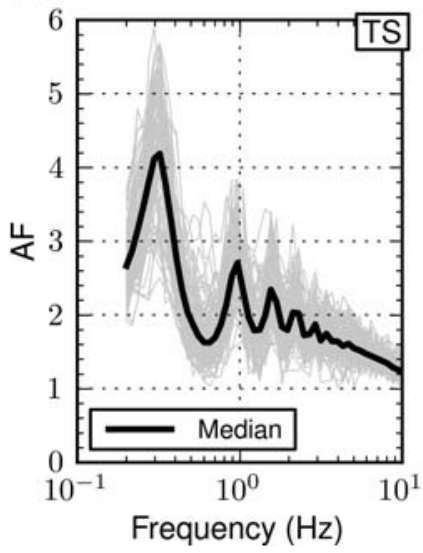

(b)

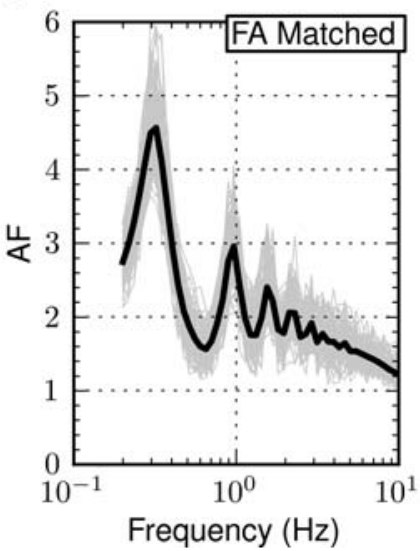

(c)

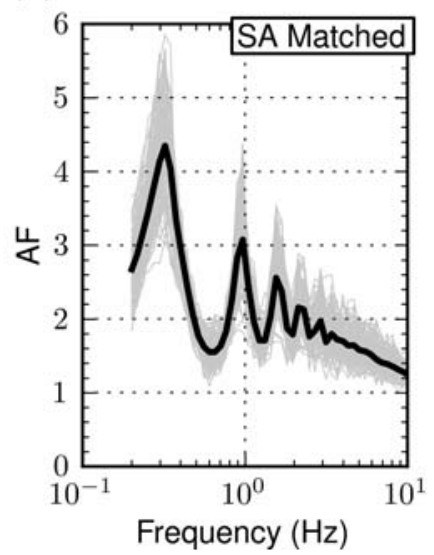

Figure 10. The influence of the input-motion variability on the amplification factors computed by TS analysis for the hypothetical site with $H_{\text {soil }}=316 \mathrm{~m}$ and $V_{S, \text { rock }}=3000 \mathrm{~m} / \mathrm{s}$.

below 3.0 for the FA- and SA-matched motions, Fig. 10), but maintaining larger predictions in amplification factor (e.g., above 5.0 in Fig. 10).

The $\alpha_{\mathrm{AF}}$ are recomputed using the amplification factors from TS analyses with the different input motions along with RVT-amplification factors corrected for duration changes. The results are plotted in Figure 11. Using the FA-matched or SA-matched motions only reduces the $\alpha_{\mathrm{AF}}$ moderately and the effect is only noticeable for the sites with lower natural frequencies (i.e., CC, hypothetical with $H_{\text {soil }}=100$ and $316 \mathrm{~m}$ ). Thus, it appears that variability in the TS-input motions does not significantly change the RVT-TS comparison, such that the minimum and maximum values of $\alpha_{\mathrm{AF}}$ still range from 0.9 to 1.2 .

\section{Influence of Soil Nonlinearity on RVT-TS Comparisons}

Equivalent-linear analyses are performed to investigate how the differences between RVT and TS analyses change with input intensity and induced strain level. Equivalentlinear RVT and TS site-response analyses are performed with the original input motions shown in Figure 3 (median PGA $=0.17 g$ ) and the same input motions scaled by 2.35 to generate a median PGA of $0.4 g$. The $\alpha_{\mathrm{AF}}$ for the EQL analyses are plotted in Figure 12 for the SCH and CC sites along with the LE results. The RVT results used in the $\alpha_{\mathrm{AF}}$ calculation for Figure 12 are not duration corrected because the previously developed duration corrections are only theoretically applicable to LE conditions. The induced shear strains are also shown in Figure 12. For the $\mathrm{SCH}$ site (Fig. 12a), as the intensity of the input motion increases, the site frequency decreases due to softening of the straincompatible properties, such that the peak in $\alpha_{\mathrm{AF}}$ shifts to a lower frequency. Additionally, the $\alpha_{\mathrm{AF}}$ at the site frequency increases from 1.06 under LE conditions to 1.11 for an input PGA of $0.40 \mathrm{~g}$ while the $\alpha_{\mathrm{AF}}$ at $100 \mathrm{~Hz}$ remains close to unity for all analyses. The induced strains from TS and RVT analy- ses (Fig. 12c) for the SCH site are very similar. For the CC site (Fig. 12b), there is little change in the site frequency with increased input intensity due to the large depth of the site predominantly controlling the site frequency. The largest changes in $\alpha_{\mathrm{AF}}$ for the CC site under different input intensities occur at frequencies greater than about $3 \mathrm{~Hz}$, where the $\alpha_{\mathrm{AF}}$ is close to 1 under LE conditions and decreases to $0.85-$ 0.95 at an input PGA of $0.40 \mathrm{~g}$. This result appears to be caused by the RVT analysis predicting somewhat larger strains (20\%-30\% larger) than the TS analysis for this site (Fig. 12d). While the levels of damping associated with the moderately larger strains are not significantly greater, when integrated over a very deep site they result in the smaller amplification factors at higher frequencies from RVT analysis.

Duration is a potential cause in the different peak strains predicted by RVT and TS analyses. Extreme value statistics (equations 1-5) are used to compute the peak strains for RVT EQL analysis. First an FAS for shear strain is computed by multiplying the input FAS for acceleration by a transfer function that relates shear strain in a layer to input acceleration. The FAS for shear strain is then used to compute the rms shear strain $\left(\gamma_{\mathrm{rms}}\right)$ using equation (1) and the appropriate peak factor using equations (2)-(5). These terms together provide the estimate of the peak shear strain. However, in all of these calculations the specified input-motion duration is used, which assumes that the shear-strain time history has the same duration characteristics as the input accelerationtime history.

To investigate the duration characteristics of the straintime histories, the results from the TS analyses are considered. Figure 13 shows an input acceleration-time history and the associated shear-strain time history computed at a depth of $700 \mathrm{~m}$ within the $\mathrm{CC}$ site. The duration and frequency-content characteristics of the shear-strain time history are very different from the input acceleration-time history. The shear-strain time history contains more lowfrequency motion and its duration $\left(D_{5-75}\right)$ is $50 \%$ larger than 
(a)

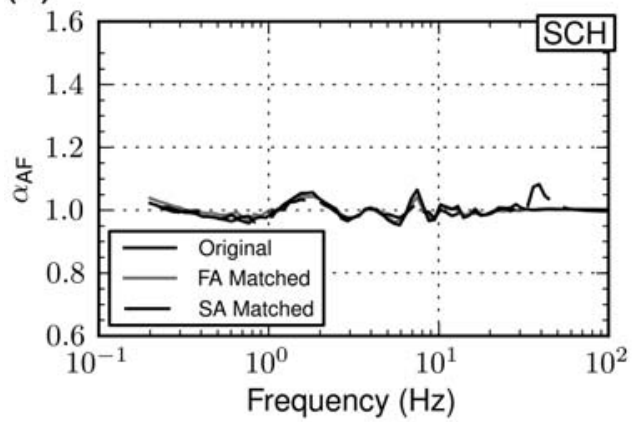

(c)

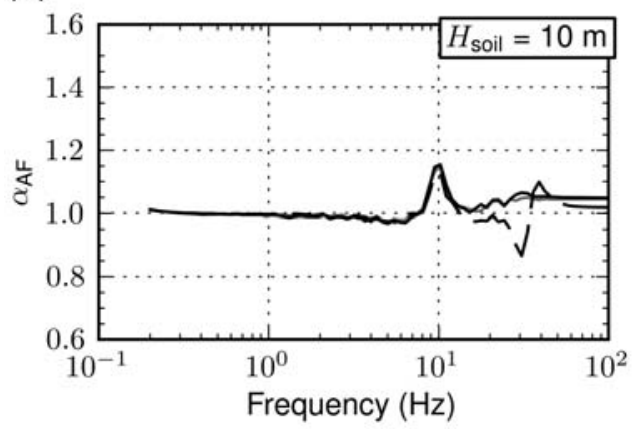

(e)

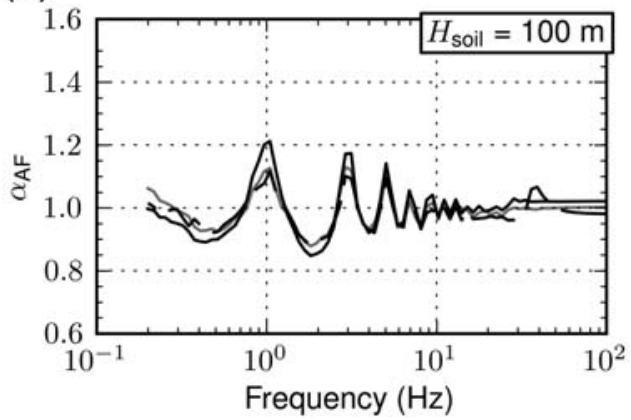

(b)

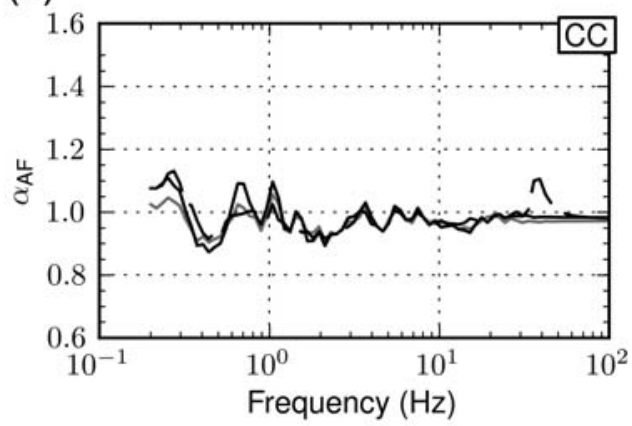

(d)

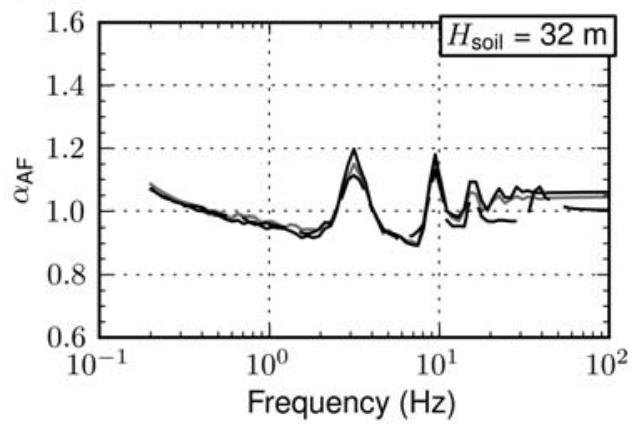

(f)

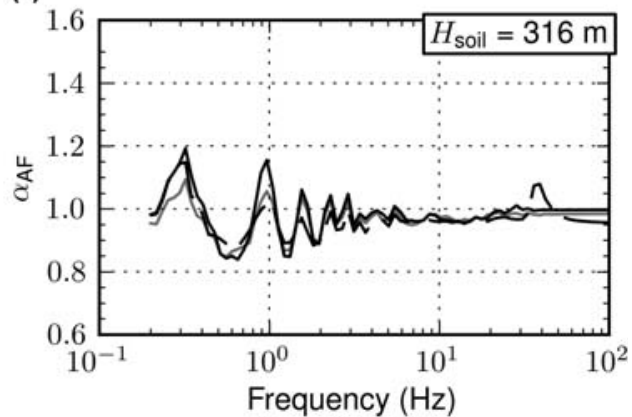

Figure 11. The influence of the input-motion variability on $\alpha_{\mathrm{AF}} \cdot \alpha_{\mathrm{AF}}$ computed from the median AF from TS analyses with different suites of input motions and the duration-corrected AF from RVT analysis.

the acceleration-time history (5.61 s versus $3.73 \mathrm{~s}$ ). The difference in frequency content for the shear-strain time history is taken into account in RVT through the shear-strain transfer function, but the duration change is not. It was also considered whether the duration of the input velocity-time history relates better to the duration of the shear-strain time history, but the duration of the velocity-time history was similar to the duration of the input acceleration-time history.

The input-motion duration $\left(D_{5-75}^{\text {input }}\right)$ and shear-strain time history durations $\left(D_{5-75}^{\text {strain }}\right)$ are investigated for different depths within the SCH and CC using the average across all motions. For the SCH site, where the TS and RVT shear strains are almost indistinguishable, the average ratio of $D_{5-75}^{\text {strain }}$ to $D_{5-75}^{\text {input }}$ is very close to 1.0 . However, the average ratio of $D_{5-75}^{\text {strain }}$ to $D_{5-75}^{\text {input }}$ for the CC site ranges from 1.6 at a depth of $700 \mathrm{~m}$ to 1.15 at a depth of $25 \mathrm{~m}$. The larger durations for strain are associated with the depths where RVT overpredicts strain.
Because the current RVT implementation uses $D_{5-75}^{\text {input }}$ in the calculation of $\gamma_{\mathrm{rms}}$ (equation 1), which is smaller than the actual $D_{5-75}^{\text {strain }}, \gamma_{\mathrm{rms}}$ is overestimated as well as the peak shear strain. Using the same approach as was applied to the amplification factors, the duration ratio can be used to correct RVT shear strain. The correction factor associated with a duration ratio of 1.6 is 0.79 , which, when applied to the RVT shear strains, makes them very similar to the TS shear strains.

\section{Influence of Site-Property Variations on RVT-TS Comparisons}

RVT site-response analyses are commonly used for seismic-hazard studies for nuclear power plants, and these studies require that site-property variability be taken into account via Monte Carlo simulation. Introducing shear-wave velocity variability reduces the peaks in the average siteamplification transfer function and because these peaks are 
(a)

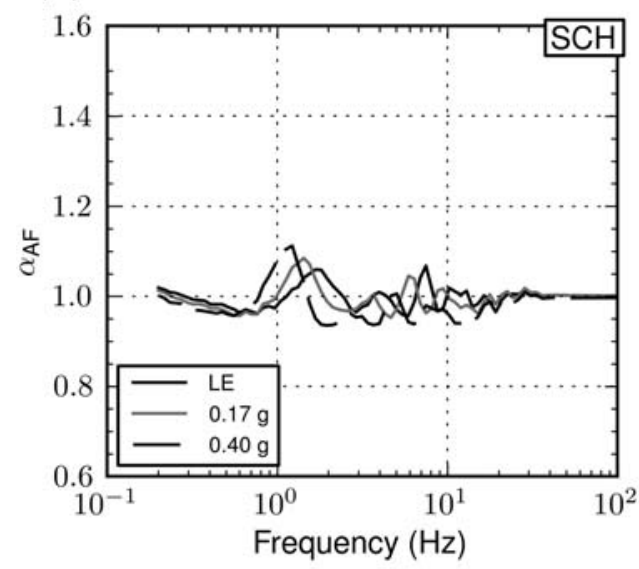

(c)

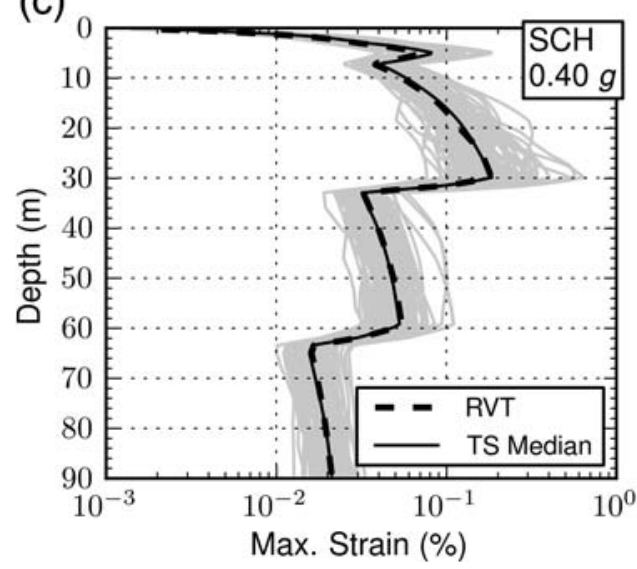

(b)
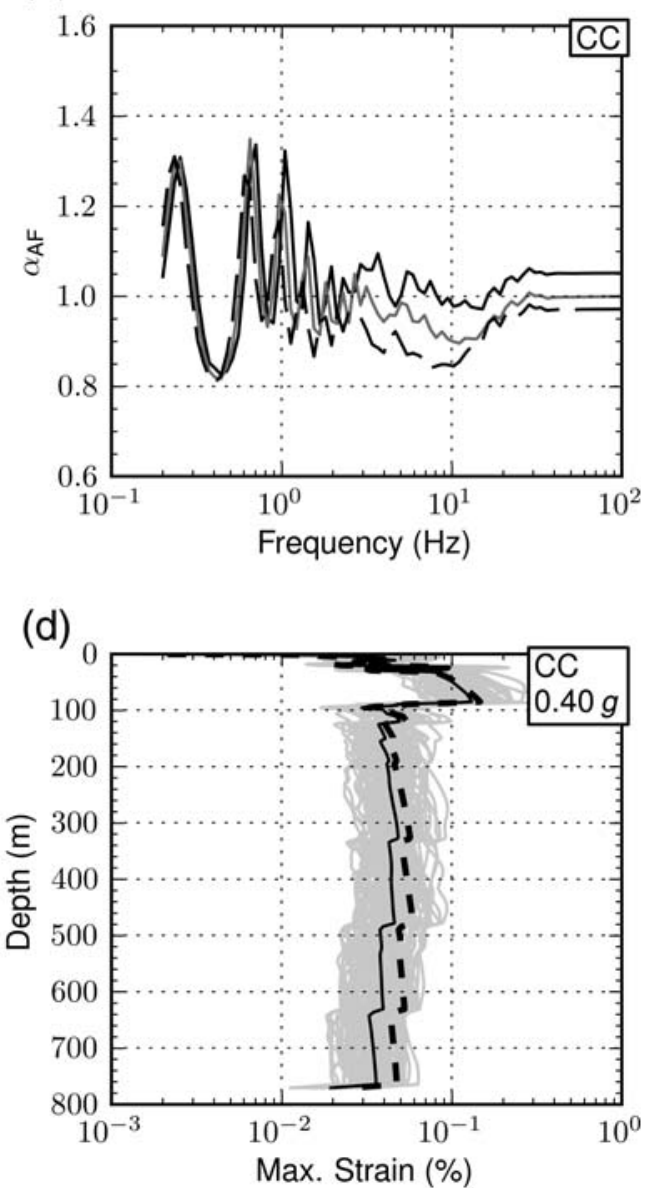

Figure 12. $(\mathrm{a}, \mathrm{b})$ The influence of input motion intensity on $\alpha_{\mathrm{AF}}$. $(\mathrm{c}, \mathrm{d})$ Peak shear-strain profiles from RVT and TS analyses and an input PGA of $0.4 g$.
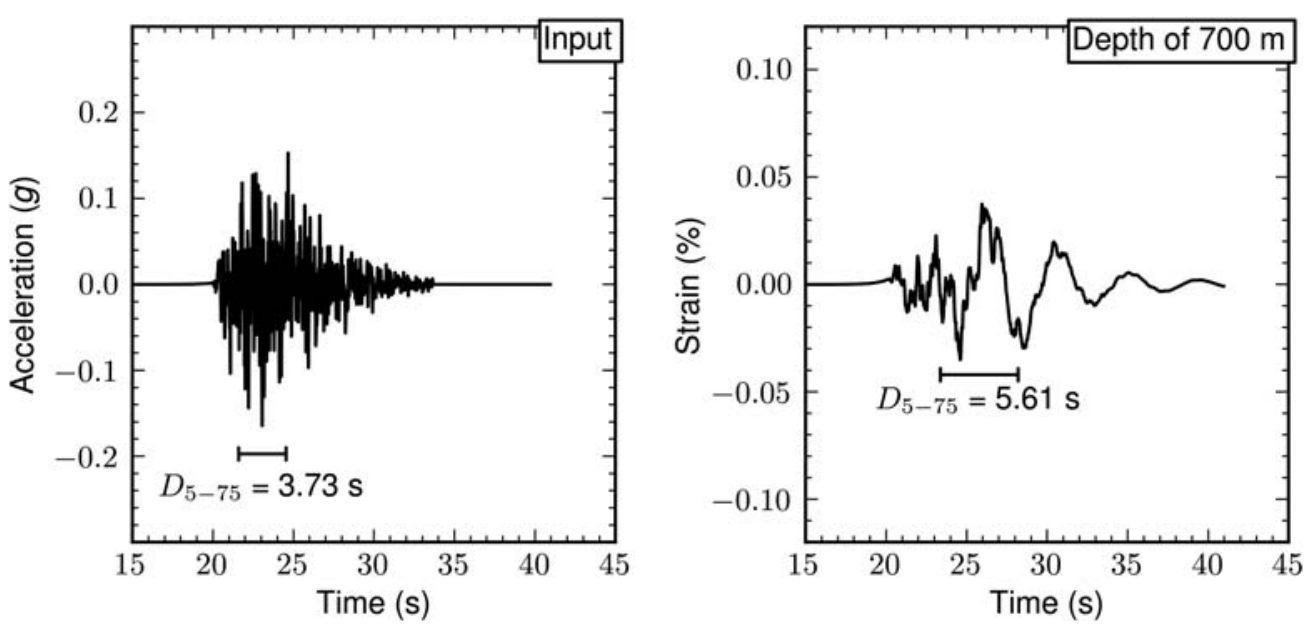

Figure 13. The input motion acceleration-time history and computed shear-strain time history at a depth of $700 \mathrm{~m}$ for the CC site.

generating the RVT overpredictions of site amplification, it is important to assess how site-property variability influences the comparison between RVT and TS site-response analyses.

RVT site-response analyses are performed with the shear-wave velocity profiles varied through Monte Carlo simulation, as incorporated in the program Strata (Kottke and Rathje, 2008). The Monte Carlo simulations simulate velocity profiles using the Toro (1995) model, which correlates the velocities in adjacent layers through an interlayercorrelation coefficient that varies with layer thickness and 

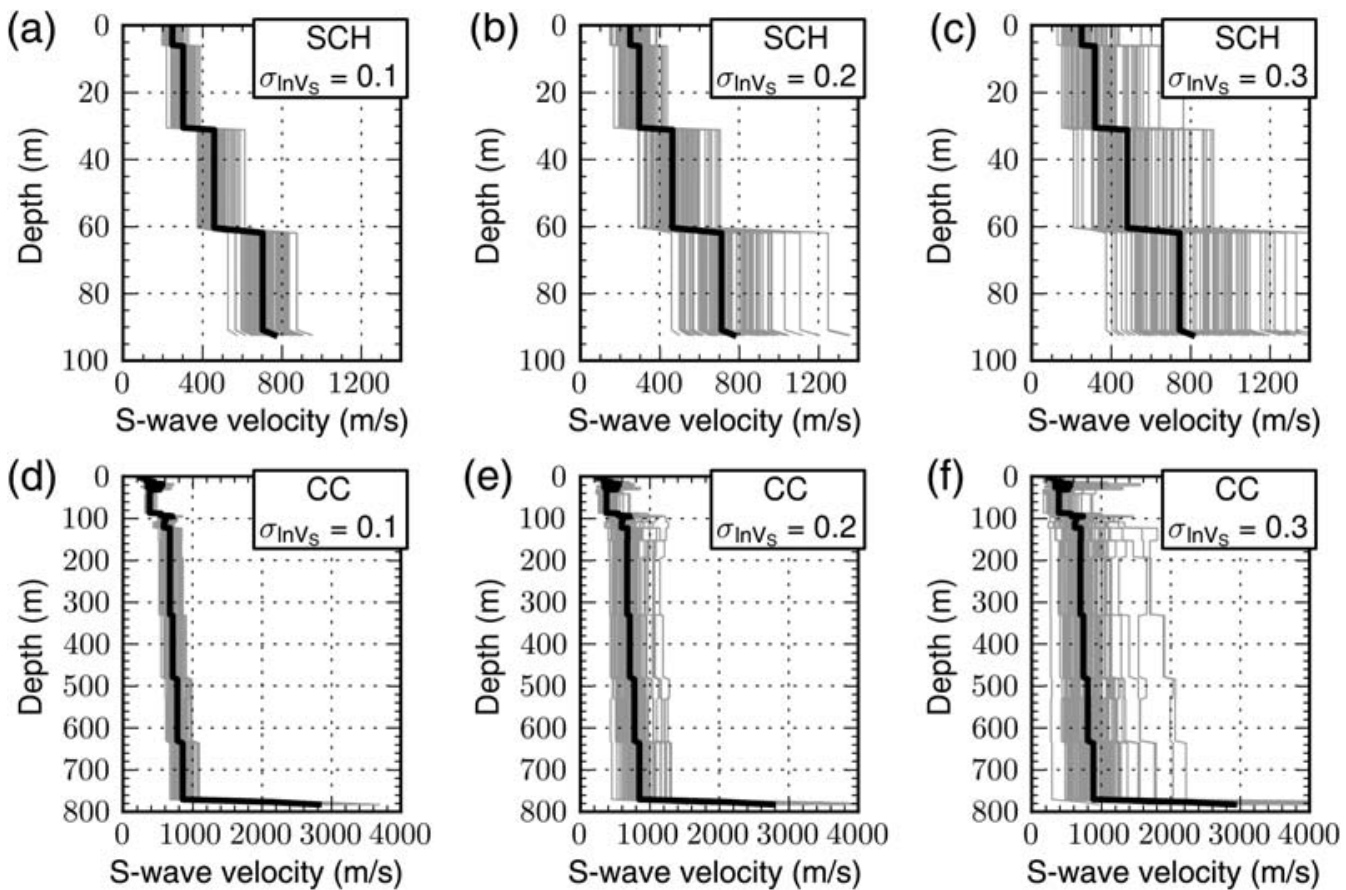

Figure 14. Shear-wave velocity profiles generated by Monte Carlo simulation for the SCH and CC sites for three different levels of shear-wave velocity variability $\left(\sigma_{\ln V_{S}}\right)$.

(a)

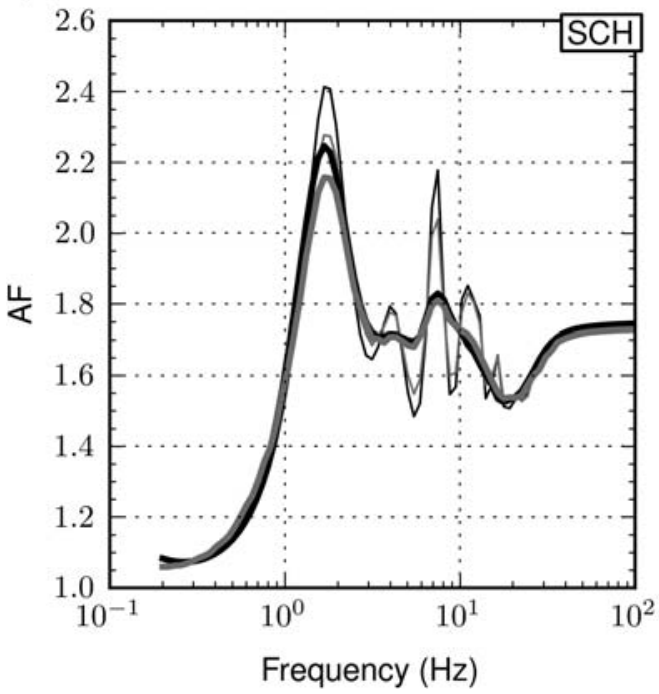

(b)

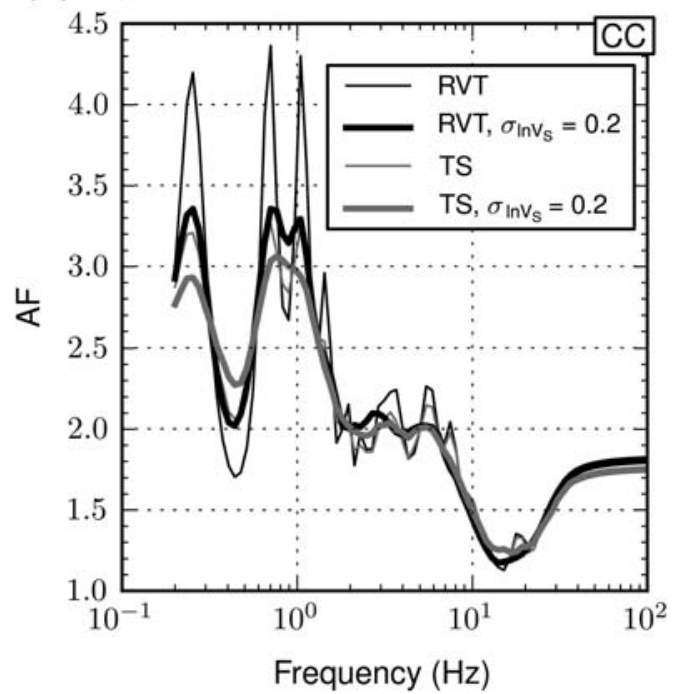

Figure 15. The influence of shear-wave velocity variability on the site amplification predicted by RVT and TS site-response analysis.

depth. The thicknesses of the layers in the baseline-velocity profiles (i.e., Fig. 1; Table 1) are maintained and velocity profiles are generated for three different levels of variability in the shear-wave velocity $\left(\sigma_{\ln V_{S}}=0.10,0.20\right.$, and 0.30$)$, as shown in Figure 14 for the $\mathrm{SCH}$ and $\mathrm{CC}$ sites. One hundred velocity-profile realizations are generated for each $\sigma_{\ln V_{S}}$ and each velocity profile is used to compute the LE site response using RVT and TS analysis. No duration corrections are applied to the RVT results.
The influence of velocity variations $\left(\sigma_{\ln V_{S}}=0.20\right)$ on the computed RVT and TS median amplification factors for the SCH and CC sites is shown in Figure 15. The median amplification factors are reduced when velocity variations are included because the velocity variations essentially move the locations of the transfer-function peaks, resulting in smaller average transfer-function peaks at the natural frequencies. However, the effect of velocity variations is more significant for the RVT results, because the peaks in the 

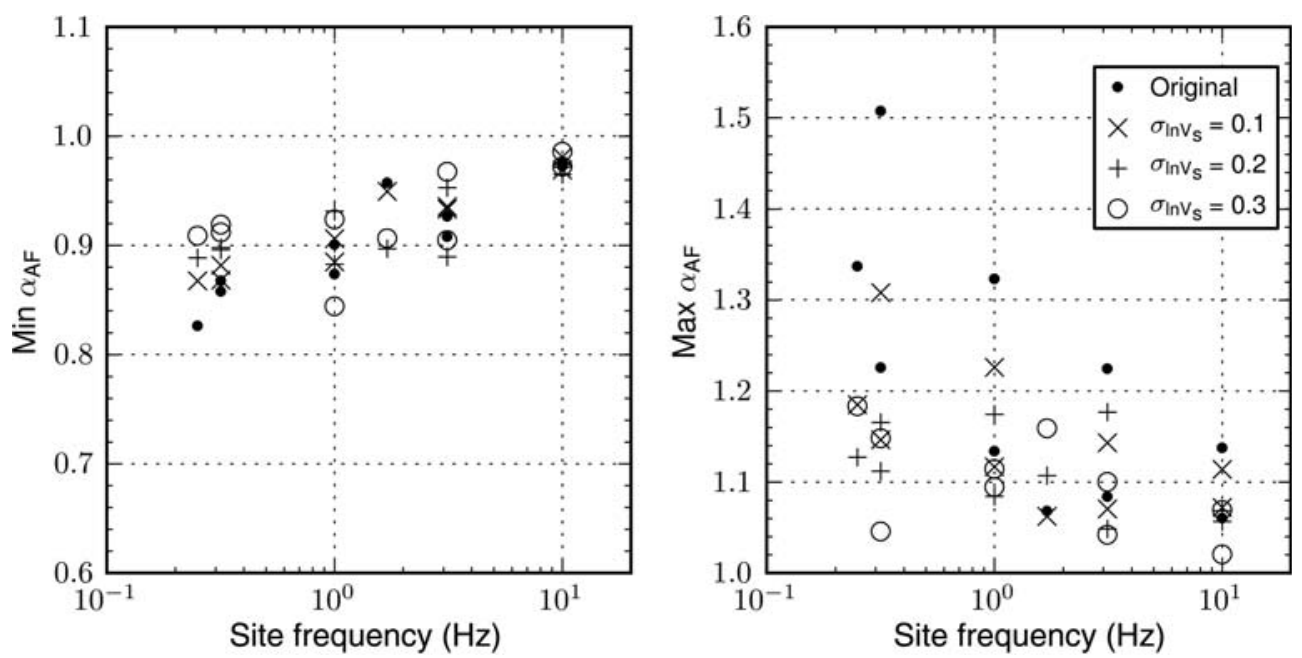

Figure 16. The influence of shear-wave velocity variability on the minimum and maximum $\alpha_{\mathrm{AF}} . \alpha_{\mathrm{AF}}$ is computed using uncorrected RVT results.

transfer function have a larger effect on the predicted RVT amplification. For the CC site, the overprediction by RVT at the first-mode frequency is reduced from about $30 \%$ to $15 \%$ when velocity randomizations with $\sigma_{\ln V_{S}}=0.20$ are used. Therefore, modeling velocity variations in the siteresponse analysis provides RVT results that are more similar to the TS results.

To evaluate the influence of site variability on the RVTTS comparison, linear-elastic RVT analyses with siteproperty variability are computed for the hypothetical sites as well as the SCH and CC sites. The minimum and maximum $\alpha_{\mathrm{AF}}$ are plotted versus site natural frequency for all sites in Figure 16. The $\alpha_{\mathrm{AF}}$ values are shown for different levels of shear-wave velocity variation (i.e., $\sigma_{\ln V_{S}}$ ). The data in Figure 16a show a moderate increase in the minimum $\alpha_{\mathrm{AF}}$ towards 1.0 for $\sigma_{\ln V_{S}}$ greater than zero, but a $10 \%-15 \%$ underprediction by RVT is still observed. The maximum $\alpha_{\mathrm{AF}}$ is influenced more by site variability than the minimum $\alpha_{\mathrm{AF}}$ (Fig. 16b). Using $\sigma_{\ln V_{S}}$ greater than zero results in a consistent improvement (i.e., reduction) in the maximum $\alpha_{\mathrm{AF}}$ such that almost all values are below 1.2 for $\sigma_{\ln V_{S}} \geq 0.2$ (Fig. 16b). The results in Figure 16 show that when shearwave velocity variability is included in site-response analyses, RVT analysis provides median-site amplification results more similar to TS analysis. The agreement improves with increasing levels of variability.

Including variability in the shear-wave velocity profile in RVT site-response analyses provides results that are in better agreement with the median predictions from TS analysis, but it does not completely resolve the differences between RVT and TS analyses. Differences as large as 10\% to $20 \%$ are still observed. These levels of difference start to approach a level that is acceptable in earthquake engineering. However, one must recognize that varying the shear-wave velocity profile introduces variability in the RVT amplification factors, and this variability may be larger than one observes in a suite of TS analyses that incorporates shear-wave velocity variability. If one is considering only the median-site amplification, then this difference in variability is not important and using Monte Carlo simulations to improve the performance of RVT site-response analysis may be a viable option. However, the increase in variability in the RVT amplification factors will influence a soil-specific seismic-hazard curve that incorporates site-specific site amplification and its variability. This issue is explored in Pehlivan et al. (2012).

\section{Discussion}

The results from this study indicate that changes in ground-motion duration due to site response, which are not taken into account in RVT site-response analysis, can lead to RVT analysis overestimating site amplification relative to TS analysis. The overprediction occurs at the natural frequencies of a site. The RVT predictions can be improved to agree better with TS analysis through a duration correction. However, this duration correction is frequency dependent and site specific (Fig. 7). One approach to developing the frequencydependent correction factors for a site would be to perform a small suite of linear-elastic TS and RVT analyses and use the results to develop the required frequency-dependent correction factors. While this approach eliminates one of the main advantages of RVT site-response analysis (i.e., no need to select input motions), the input motions could be generated quickly via the stochastic method and used to develop the corrections factors. Alternatively, because the frequency dependence and amplitude of the correction factors are related to the transfer function for the site, an empirical relationship could be developed that predicts the correction factors as a function of the linear-elastic transfer function for a site. This empirical relationship could be developed from correction factors computed for a large range of sites with different characteristics. This approach would avoid the need 
for users to perform TS analyses to correct their RVT analyses, but the correction factors would be less accurate than those developed on a site-specific basis.

Another required improvement to RVT site response relates to the duration used in the shear-strain calculation. Using the input-motion duration in the RVT calculation of shear strain can overestimate the induced shear strains, leading to overdamping of the high-frequency components of motions for very deep sites and/or large strains. An adjustment to the input-motion duration that makes it representative of a shear-strain time history can improve the RVT shear-strain calculation. Again, this adjustment is site specific and could be estimated from TS analyses. Alternatively, an empirical relationship could be developed that predicts the adjustment factor based on site characteristics.

The corrections proposed here are most necessary for RVT site-response analyses that use deterministic soil properties. When RVT site-response analyses are used within a Monte Carlo framework, in which the shear-wave velocity profile is statistically varied and the site amplification is averaged over the shear-wave velocity realizations, the differences between RVT and TS analyses are more modest (Fig. 16). While correction factors would further improve the comparison between RVT and TS analyses when site property variability is modeled, these correction factors may not be as critical as for analyses with deterministic soil properties.

\section{Conclusions}

The RVT approach to site-response analysis has been used in seismic-hazard studies since the 1990s. It is an attractive alternative to site-response analyses using TS input motions because a single RVT analysis can potentially provide the mean site response without the need for a suite of input-time histories. RVT site-response analysis is commonly used in seismic-hazard studies for nuclear power plants because these studies often include numerous site-response simulations that account for variability in the site characteristics. Recent work by Rathje and Ozbey (2006) identified the potential for RVT to overpredict site amplification relative to TS analyses. The present article extends the work of Rathje and Ozbey (2006) to better elucidate the differences between RVT and TS site-response analyses for a range of site conditions.

The site-response results presented in this paper indicate that RVT consistently overpredicts the site amplification at the fundamental frequencies of a site as compared with the median value from a suite of input-time series. This overprediction is modest (i.e., less than $10 \%$ ) for sites with natural frequencies greater than about $1 \mathrm{~Hz}$ and with a soft rock (i.e., $V_{S \text {,rock }} \sim 1000 \mathrm{~m} / \mathrm{s}$ ) half-space, but the overprediction can be as large as $50 \%$ for sites with low natural frequencies (i.e., $0.2-0.3 \mathrm{~Hz}$ ) and a hard rock (i.e., $V_{S \text {,rock }} \sim 3000 \mathrm{~m} / \mathrm{s}$ ) half-space. There is some underprediction of site amplification at frequencies between the fundamental modes, but the underprediction is relatively modest $(5 \%-15 \%)$. The overpredicted response by RVT is principally caused by a change in the duration of the ground motion and associated oscillator response generated by the site response. Ignoring the increase in duration due to site response causes the $a_{\text {rms }}$ acceleration to be overestimated by RVT and thus the peak acceleration and spectral acceleration are overestimated as well. Correcting the RVT spectral accelerations for this duration increase using the observed TS durations brings the RVT estimates within $10 \%$ of the TS values for sites with $V_{S \text {,rock }} \sim 1000 \mathrm{~m} / \mathrm{s}$ and within $20 \%$ for sites with $V_{S, \text { rock }} \sim 3000 \mathrm{~m} / \mathrm{s}$.

RVT site-response analysis also tends to overpredict shear strains relative to TS analysis predominantly for deep sites (i.e., smaller natural frequencies) underlain by hard rock. Even a modest overestimation of shear strain in equivalent-linear site-response analysis can result in overdamping of high-frequency spectral accelerations when the larger strains extend over significant depths. This overestimation again is driven by changes in duration. The duration of the shear-strain time history is increased by the site response and this increase currently is not taken into account by RVT analysis.

When shear-wave velocity variability is incorporated into the site-response analysis via Monte Carlo simulation, the overprediction of site amplification by RVT is reduced from as much as $30 \%-50 \%$ to about $10 \%-20 \%$. The improved agreement between RVT and TS analyses observed when shear-wave velocity variability is modeled is due to the variability reducing the amplitudes of the peaks in the average transfer function, which is the main cause for the RVT overprediction in site response. Nonetheless, there are still consistent differences between the RVT and TS results.

This paper focused on identifying systematic differences between RVT and TS site-response analyses and explaining these differences. An important finding is that changes to the durations of ground shaking, oscillator response, and shear strains due to site response influence the differences between RVT and TS results. Corrections for these durations were estimated from the TS analyses and improved the agreement between RVT and TS analyses. A similar approach could be used in practice to improve RVT site-response predictions or empirical relationships could be developed that predict the correction factors based on the characteristics of a site.

\section{Data and Resources}

The time series and random-vibration theory siteresponse simulations performed in this study were conducted using Strata, which can be obtained from http://nees.org/ resources/strata (last accessed April 2012). The stochastic time series used in the analysis were created using the latest version of SMSIM by Boore (2003) and can be obtained from http://daveboore.com/software_online.html (last accessed January 2012). Time-domain spectral matching of the input motions was performed using the program 
RSPMATCH, which is described in Hancock (2006) and available from the authors. Examination of the results and creation of the figures was done using Python (http://python .org, last accessed December 2012) in conjunction with the numpy (http://www.numpy.org/, last accessed December 2012), scipy (http://www.scipy.org), and matplotlib (http:// matplotlib.sourceforge.net/, last accessed December 2012) libraries.

\section{Acknowledgments}

Financial support was provided by the Nuclear Regulatory Commission under grant NRC-04-07-122. This support is gratefully acknowledged. We thank David Boore, Frank Scherbaum, Steven Day, and an anonymous reviewer for thoughtful comments and suggestions that improved the manuscript.

\section{References}

Atkinson, G. M., and D. M. Boore (2006). Earthquake ground-motion prediction equations for Eastern North America, Bull. Seismol. Soc. Am. 96, no. 6, 2181-2205.

Boore, D. M. (1983). Stochastic simulation of high-frequency ground motions based on seismological models of the radiated spectra, Bull. Seismol. Soc. Am. 73, no. 6A, 1865.

Boore, D. M. (2003). Simulation of ground motion using the stochastic method, Pure Appl. Geophys. 160, no. 3, 635-676.

Boore, D. M. (2005). SMSIM-Fortran programs for simulating ground motions from earthquakes: version 2.3, U.S. Geol. Surv. Open-File Rept. OFR 96-80-A, Menlo Park, California.

Boore, D. M., and W. B. Joyner (1984). A note on the use of random vibration theory to predict peak amplitudes of transient signals, Bull. Seismol. Soc. Am. 74, no. 5, 2035-2039.

Boore, D. M., and E. M. Thompson (2012). Empirical improvements for estimating earthquake response spectra with random vibration theory, Bull. Seismol. Soc. Am. 102, no. 2, 761-772.

Cartwright, D. E., and M. S. Longuet-Higgins (1956). The statistical distribution of the maxima of a random function, Proc. Roy. Soc. Lond. Math. Phys. Sci. 237, no. 1209, 212-232.

Darendeli, M. B. (2001). Development of a new family of normalized modulus reduction and material damping curves, Ph.D. Thesis, The University of Texas, Austin.

Electric Power Research Institute (EPRI) (1993). Guidelines for Determining Design Basis Ground Motions, Electric Power Research Institute, Palo Alto, California.

Gibbs, J., J. Tinsley, D. Boore, and W. Joyner (1999). Seismic velocities and geological conditions at twelve sites subjected to strong ground motion in the 1994 Northridge, California, earthquake: a revision of OFR 96740, U.S. Geological Survey Open-File Rept. 99-446, U.S. Department of the Interior, U.S. Geological Survey, Menlo Park, California.
Hancock, J. (2006). An improved method of matching response spectra of recorded earthquake ground motion using wavelets, J. Earthq. Eng. 10, no. 1, 67-89.

Hanks, T. C., and R. K. McGuire (1981). The character of high-frequency strong ground motion, Bull. Seismol. Soc. Am. 71, no. 6, 2071.

Kottke, A. R., and E. M. Rathje (2008). Technical Manual for Strata, PEER Report 2008/10, Pacific Earthquake Engineering Research Center, University of California at Berkeley, February, 84 pp.

Liu, L., and S. Pezeshk (1999). An improvement on the estimation of pseudo-response spectral velocity using RVT method, Bull. Seismol. Soc. Am. 89, no. 5, 1384-1389.

McGuire, R. K., A. M. Becker, and N. C. Donovan (1984). Spectral estimates of seismic shear waves, Bull. Seismol. Soc. Am. 74, no. 4, 1427-1440.

Nuclear Regulatory Commission (NRC) (2007). Regulatory Guide 1.208: Performance-Based Approach to Define Site-Specific Earthquake Ground Motion, United States Nuclear Regulatory Commission, Washington, DC.

Pehlivan, M., E. M. Rathje, and R. B. Gilbert (2012). Incorporating sitespecific site response analysis into PSHA, Second Internal Conference on Performance-Based Design in Earthquake Geotechnical Engineering, Taormina, Italy.

Rathje, E. M., and M. C. Ozbey (2006). Site-specific validation of random vibration theory-based seismic site response analysis, J. Geotech. Geoenv. Eng., 132, no. 7, 911-922.

Schnabel, P. B., H. B. Seed, and J. B. Lysmer (1972). SHAKE: A computer program for earthquake response analysis on horizontally layered sites, Report No. UCB/EERC-72/12, EERI University of California, Berkeley, California.

Silva, W. J., N. A. Abrahamson, G. Toro, and C. Costantino (1997). Description and validation of the stochastic ground motion model, Report for Brookhaven National Laboratory, Upton, New York.

Toro, G. R. (1995). Probabilistic models of site velocity profiles for generic and site-specific ground-motion amplification studies, Technical Report 779574, Brookhaven National Laboratory, Upton, New York.

Pacific Earthquake Engineering Research (PEER) Center

325 Davis Hall

University of California

Berkeley, CA 94720-1792

albert.kottke@gmail.com

(A.R.K.)

University of Texas at Austin

Department of Civil, Architectural, and Environmental Engineering

301 E. Dean Keeton St, Stop C1700

Austin, Texas 78712

e.rathje@mail.utexas.edu

(E.M.R.)

Manuscript received 10 August 2012 